Pacific

Journal of

Mathematics

\title{
VALUATIVE MULTIPLIER IDEALS
}

ZHENGYU HU 


\title{
VALUATIVE MULTIPLIER IDEALS
}

\author{
ZHENGYU HU
}

\begin{abstract}
The main goal of this paper is to construct an algebraic analogue of quasiplurisubharmonic function (qpsh for short) from complex analysis and geometry. We define a notion of qpsh function on a valuation space associated to a quite general scheme. We then define the multiplier ideals of these functions and prove some basic results about them, such as subadditivity property, the approximation theorem. We also treat some applications in complex algebraic geometry.
\end{abstract}

\section{Introduction}

Given a line bundle $L$ on a smooth projective complex variety, a classical theorem of Kodaira asserts that if $L$ carries on a smooth metric with positive curvature, then $L$ is ample; equivalently, the global sections of a multiple of $L$ give an embedding to a projective space and hence induce such a metric on $L$. More generally, global sections of a multiple of $L$ induce a semipositive singular metric. Conversely, given a semipositive singular metric $h$, the local weight function $\varphi$, which is plurisubharmonic (psh for short), should be related to sections of multiples of $L$, or perhaps of a small perturbation of $L$. See [Lehmann 2011] for more details.

On the other hand, if we work locally near the origin of $\mathbb{C}^{n}$, then Section 5 of [Boucksom et al. 2008] shows that we can transform a psh germ $\varphi$ to a formal psh function $\widehat{\varphi}$ on quasimonomial valuations centred at the origin. This valuative transform usually loses much information on the original psh function, however, it preserves the information on the singularity of $\varphi$. In particular, they give the same multiplier ideals which essentially means that they characterize the same singularity because of the Demailly's approximation. The idea of studying psh functions using valuations was systematically developed in the work just cited and its predecessors [Favre and Jonsson 2004; 2005a; 2005b]. The main purpose of this paper is to define a similar notion of qpsh functions on a separated, regular, connected and excellent schemes over $\mathbb{Q}$, and we then study these functions.

Although we don't discuss Berkovich spaces in this paper, our work should be related to the qpsh functions (or metrics on line bundles) on the Berkovich 
space associated to a smooth projective variety over a trivially valued field. See [Boucksom et al. 2012b; 2012c].

Let us briefly introduce some terminology. Roughly speaking, we consider a function $\varphi$ on divisorial valuations on a scheme $X$ such that $\varphi\left(t \operatorname{ord}_{E}\right)=t \varphi\left(\operatorname{ord}_{E}\right)$ and $\sup _{E}\left|\varphi\left(\operatorname{ord}_{E}\right)\right| / A\left(\operatorname{ord}_{E}\right)<+\infty$, where $E$ runs over all prime divisors over $X$. We prove that such functions form a $\mathrm{Banach}$ space $\mathrm{BH}(X)$ if we equip it with the norm $\|\varphi\|=\sup _{E}\left|\varphi\left(\operatorname{ord}_{E}\right)\right| / A\left(\operatorname{ord}_{E}\right)$ (see Proposition 3.2). By convention we set $\log |\mathfrak{a}|\left(\operatorname{ord}_{E}\right)=-\operatorname{ord}_{E}(\mathfrak{a})$ for a nonzero coherent ideal sheaf $\mathfrak{a}$, and one can easily check that $\log |\mathfrak{a}|$ is a valuative function in $\mathrm{BH}(X)$. We define the set of qpsh functions QPSH $(X)$ to be the closed convex cone generated by functions of the form $\log |\mathfrak{a}|$. We then define the multiplier ideal $\mathscr{E}(\varphi)$ of a qpsh function $\varphi$ to be the largest ideal $\mathfrak{a}$ such that $\sup _{E}\left(-\operatorname{ord}_{E}(\mathfrak{a})-\varphi\left(\operatorname{ord}_{E}\right)\right) / A\left(\operatorname{ord}_{E}\right)<1$. This definition is reasonable because of Proposition 4.3 and Corollary 4.14.

Our first main result is that a qpsh function is a decreasing limit of a sequence of qpsh functions of the form $c_{k} \log \left|\mathfrak{b}_{k}\right|$. In complex analysis and geometry, such a regularization is crucial. See [Demailly 1992; 1993]. Moreover, we prove that we can actually choose $\mathfrak{b}_{k}=\mathscr{g}(k \varphi)$ satisfying the subadditivity property. See Proposition 4.22(1). Readers can compare this result with [Demailly et al. 2000].

Theorem 1.1 (cf. Theorem 4.24). Let $\varphi$ be a bounded homogeneous function. Then $\varphi$ is qpsh if and only if $\varphi$ is the limit function, in norm, of a decreasing sequence of qpsh functions of the form $c_{k} \log \left|\mathfrak{b}_{k}\right|$. Furthermore, we can choose $c_{k}=1 / k$ and $\mathfrak{b}_{k}=\mathscr{g}(k \varphi)$ which form a subadditive sequence of ideals.

Given an ideal $\mathfrak{a}$ on a scheme $X$, the $\log$ canonical threshold $\operatorname{lct}(\mathfrak{a})$ is a fundamental invariant both in singularity theory and birational geometry (see [Lazarsfeld 2004; Kollár and Mori 1998], etc.). The log canonical threshold admits the following description in terms of valuations:

$$
\operatorname{lct}(\mathfrak{a})=\inf _{E} \frac{A\left(\operatorname{ord}_{E}\right)}{\operatorname{ord}_{E}(\mathfrak{a})}
$$

where $E$ runs over all prime divisors over $X$ and $A\left(\operatorname{ord}_{E}\right)=\operatorname{ord}_{E}\left(K_{Y / X}\right)+1$. In fact in the above formulae one can take the infimum over all real valuations centred on $X$. It is well-known that if $Y$ is a $\log$ resolution of $\mathfrak{a}$, then there exists some prime divisor $E$ on $Y$ such that $\operatorname{ord}_{E}$ computes the $\log$ canonical threshold, that is, $\operatorname{lct}(\mathfrak{a})=A\left(\operatorname{ord}_{E}\right) / \operatorname{ord}_{E}(\mathfrak{a})$. Given a qpsh function $\varphi$, we can define the log canonical threshold $\operatorname{lct}(\varphi)$ as the limit of $1 / c_{k} \operatorname{lct}\left(\mathfrak{a}_{k}\right)$, where $c_{k} \log \left|\mathfrak{a}_{k}\right|$ converges to $\varphi$ strongly in norm. We show that

$$
\operatorname{lct}(\varphi)=\inf _{E} \frac{A\left(\operatorname{ord}_{E}\right)}{-\varphi\left(\operatorname{ord}_{E}\right)}
$$


Unfortunately, there might be no divisorial valuation that computes the log canonical threshold in general. However, we can prove that there exists a real valuation that computes the log canonical threshold. This has been heavily studied in [Jonsson and Mustață 2012; 2014] and other references. Conjecture B of [Jonsson and Mustață 2012] suggests that a valuation that computes the norm is quasimonomial (see Conjecture 5.9). Equivalently we consider the reciprocal of the log canonical threshold, which is exactly the norm of $\varphi$ by definition. More generally, for a nonzero ideal $\mathfrak{q}$ we consider $\|\varphi\|_{\mathfrak{q}}:=\sup _{E}\left(-\varphi\left(\operatorname{ord}_{E}\right) /\left(A\left(\operatorname{ord}_{E}\right)+\operatorname{ord}_{E}(\mathfrak{q})\right)\right.$, and we prove that there exists a real valuation that computes this norm. The proof in this paper mainly follows the strategy of [Jonsson and Mustață 2012]. A similar result appears in [Jonsson and Mustață 2014].

Theorem 1.2 (Theorem 5.2). Let $\varphi \in \mathrm{QPSH}(X)$ be a qpsh function and let $\mathfrak{q}$ be a nonzero ideal on $X$. Then there exists a nontrivial tempered valuation $v$ that computes $\|\varphi\|_{\mathfrak{q}}$.

If $X$ is a complex projective variety, then we can provide $\operatorname{QPSH}(X)$ with more structures. Namely, given a $\mathbb{Q}$-line bundle $L$ on $X$, we say that the function $\lambda \log |\mathfrak{a}|$ is $L$-psh if $\lambda$ is a nonnegative rational number and $L \otimes \mathfrak{a}^{\lambda}$ is semi-ample. We can then define $\operatorname{PSH}(L) \subseteq \mathrm{QPSH}(X)$ as the closure of the set of such functions. We also define the set of pseudo $L$-psh functions as $\operatorname{PSH}_{\sigma}(L):=\bigcap_{\epsilon>0} \operatorname{PSH}(L+\epsilon A)$, where $A$ is an ample line bundle. See the section on $D$-psh functions (page 118) for more details.

In this setting, we show that there exists the maximal $L$-psh function $\varphi$ that can be written explicitly as $\varphi(v)=-v(\|L\|)$, and that there exists the maximal pseudo $L$-psh function $\phi$ that can be written explicitly as $\phi(v)=-\sigma_{v}(\|L\|)$ (see Propositions 6.10 and 6.11). As an immediate corollary we generalize Theorem 6.14 of [Lehmann 2011] as follows (see that paper for the definitions of the perturbed ideal and the diminished ideal).

Theorem 1.3 (Theorem 6.16). Let D be a pseudo-effective divisor. Assume that $\phi_{\max }$ is the maximal pseudo D-psh function. Then the perturbed ideal and the diminished ideal are $\mathscr{F}_{\sigma,-}(D)=\mathscr{F}_{-}\left(\phi_{\max }\right)$ and $\mathscr{F}_{\sigma}(D)=\mathscr{g}\left(\phi_{\max }\right)$, respectively. In particular, we can write $\mathscr{F}_{\sigma}(D)$ explicitly as

$$
\Gamma\left(U, \mathscr{g}_{\sigma}(L)\right)=\left\{f \in \Gamma\left(U, O_{X}\right) \mid v(f)+A(v)-\sigma_{v}(\|L\|)>0 \text { for all } v \in \mathrm{V}_{U}^{*}\right\} .
$$

Further, a nonzero ideal $\mathfrak{q} \subseteq \mathscr{F}_{\sigma}(\|L\|)$ if and only if $v(\mathfrak{q})+A(v)-\sigma_{v}(\|L\|)>0$ for all $v \in \mathrm{V}_{X}^{*}$.

In the last subsection of this paper, we prove the finite generation of a divisorial module as another application. The proposition below can also be obtained using minimal model theory (see Remark 6.21). Note that our proof here avoids using "the length of extremal rays" (see [Birkar and Hu 2012]). 
Proposition 1.4 (Proposition 6.18). Let $(X, B)$ be a log canonical pair. Assume that $K_{X}+B$ is $\mathbb{Q}$-Cartier and abundant, and that $R\left(K_{X}+B\right)$ is finitely generated. Then, if $\mathscr{F}$ is any reflexive sheaf, $M_{\mathscr{F}}^{p}\left(K_{X}+B\right)$ is a finitely generated $R\left(K_{X}+B\right)$-module.

This proposition can be slightly generalized (see Proposition 6.24).

\section{Valuation spaces}

Throughout this paper, all schemes are assumed to be separated, regular, connected and excellent schemes over $\mathbb{Q}$. All rings are assumed to be integral, regular and excellent rings containing $\mathbb{Q}$. An ideal on a scheme means a coherent ideal sheaf on a scheme. A birational model of a scheme is a model birational to and proper over this scheme, and a divisor over a scheme is a divisor on a birational model of the scheme. For definitions and properties of valuations, multiplier ideals, singularities in birational geometry, etc., see [Kollár and Mori 1998; Lazarsfeld 2004; Jonsson and Mustaţă 2012]. From now on we abbreviate this last reference as [JM12].

Real valuations. Let $X$ be a scheme, and let $K(X)$ be its function field. A real valuation $v$ is a function $v: K(X)^{*} \rightarrow \mathbb{R}$ such that $v(f g)=v(f)+v(g)$ and $v(f+g) \geq \min \{v(f), v(g)\}$. By convention we set $v(0):=+\infty$. Let

$$
\mathrm{O}_{v}:=\{f \mid v(f) \geq 0\}
$$

be its valuation ring. If there exists a point $\xi \in X$ such that the morphism $\mathrm{O}_{X, \xi} \hookrightarrow \mathrm{O}_{v}$ is a local homomorphism, then $\xi$ is called the centre of $v$ on $X$ and denoted by $c_{X}(v)$. Note that $\xi$ is unique since $X$ is separated, and also note that the centre always exists provided that $X$ is complete. A real valuation with centred on $X$ is called a real valuation on $X$ or simply a valuation on $X$, and we denote by $\operatorname{Val}_{X}$ the set of valuations on $X$. The set of valuations $\operatorname{Val}_{X}$ is independent of the choice of a birational model of $X$. More precisely, if $Y \rightarrow X$ is a proper birational morphism of schemes, then $\operatorname{Val}_{X}=\operatorname{Val}_{Y}$. A valuation $v$ on $X$ is said to be the trivial valuation if its centre $c_{X}(v)$ is the generic point of $X$. We denote by $\mathrm{Val}_{X}^{*} \subseteq \mathrm{Val}_{X}$ the set of nontrivial valuations on $X$.

The set $\operatorname{Val}_{X}$ can be equipped with an induced topology defined by the maps $v \rightarrow v(f)$ for all rational functions $f \in K(X)^{*}$. For every nonzero ideal $\mathfrak{a}$, we have that $v(\mathfrak{a})$ is well defined and $v(\mathfrak{a})=v(\overline{\mathfrak{a}})$, where $\overline{\mathfrak{a}}$ denotes the integral closure of $\mathfrak{a}$. Note that the topology on $\mathrm{Val}_{X}$ defined by pointwise convergence on ideals on $X$ is equivalent to that on functions in $K(X)$. Readers can consult [JM12, Section 1] for more details.

In this topology, the map $c_{X}: \operatorname{Val}_{X} \rightarrow X$ is anti-continuous. That is, the inverse image of an open subset is closed. More precisely, if $U \subseteq X$ is an open subset and $\mathfrak{m}$ is the defining ideal of $X \backslash U$, then $\operatorname{Val}_{U}=\left\{v \in \operatorname{Val}_{X} \mid v(\mathfrak{m})=0\right\}$ and $\operatorname{Val}_{U}$ is closed in $\mathrm{Val}_{X}$. 
For two valuations $v, w$ on $X$, we say that $v \leq w$ if $v(\mathfrak{a}) \leq w(\mathfrak{a})$ for every nonzero ideal $\mathfrak{a}$. This is equivalent to that the centre $\eta:=c_{X}(w) \in \overline{c_{X}(v)}$ and that $v(f) \leq w(f)$ for every nonzero local function $f \in \mathfrak{O}_{X, \eta}$.

Quasimonomial valuations. Let $X$ be a scheme, let $\xi \in X$ be a point, and let $\underline{x}=\left(x_{1}, \ldots, x_{r}\right)$ be a regular system of parameters at $\xi$. If $f \in \mathbb{O}_{X, \xi}$ is a local regular function, then $f$ can be expressed as $f=\sum_{\beta} c_{\beta} x^{\beta}$ in $\widehat{O_{X, \xi}}$ with each coefficient $c_{\beta}$ either zero or a unit. For each $\alpha=\left(\alpha_{1}, \ldots, \alpha_{r}\right) \in \mathbb{R}_{>0}^{r}$, we define a real valuation by $\operatorname{val}_{\xi, \alpha}(f)=\min \left\{\langle\alpha, \beta\rangle \mid c_{\beta} \neq 0\right\}$, where $\langle\alpha, \beta\rangle:=\sum_{i} \alpha_{i} \beta^{i}$, which is called a monomial valuation on $X$.

A pair $(Y, D)$ is called log smooth if $Y$ is a scheme and $D$ is a reduced divisor whose components are regular subschemes intersecting each other transversally. A pair $(Y, D)$ is called a log resolution of $X$ if there is a birational projective morphism $\pi: Y \rightarrow X$ and $\left(Y, D+K_{Y / X}\right)$ is $\log$ smooth. Let $\left(Y^{\prime}, D^{\prime}\right)$ be another $\log$ resolution of $X$, we say $\left(Y^{\prime}, D^{\prime}\right) \succeq(Y, D)$ if $Y^{\prime}$ is projective over $Y$ and the support of $D^{\prime}$ contains the support of the pullback of $D$. Note that $\log$ resolutions of $X$ form an inverse system.

Let $(Y, D)$ be a log resolution of $X$, and let $\eta$ be the generic point of an irreducible component of the intersection of some prime components of $D$. We denote by $\mathrm{QM}_{\eta}(Y, D)$ the set of real valuations which can be defined as a monomial valuation at $\eta$. Note that $\eta \in \overline{c_{X}(v)}$ and $\mathrm{QM}_{\eta}(Y, D) \cong \mathbb{R}_{\geq 0}^{r}$ as topological spaces. We also define

$$
\mathrm{QM}(Y, D)=\bigcup_{\eta} \mathrm{QM}_{\eta}(Y, D),
$$

where $\eta$ runs over every generic point of some component of the intersection of some prime components of $D$. A real valuation $v$ is said to be quasimonomial if there exists a $\log$ resolution $(Y, D)$ such that $v \in \mathrm{QM}(Y, D)$.

Remark 2.1. Let $\Gamma_{v}=v\left(K(X)^{*}\right) \subseteq \mathbb{R}$ be the value group of $v$. Denote by $\operatorname{ratrk}(v)=$ $\operatorname{dim}_{\mathbb{Q}}\left(\Gamma_{v} \otimes_{\mathbb{Z}} \mathbb{Q}\right)$ the rational rank of $v$, and let $k_{v}, k(\xi)$ be the residue fields of $\mathrm{O}_{v}$, $\mathcal{O}_{X, \xi}$ respectively, where $\xi=c_{X}(v)$. If we let $\operatorname{trdeg}_{X}(v)=\operatorname{trdeg}\left(k_{v} / k(\xi)\right)$ be the transcendental degree of $v$ over $X$, we have Abhyankar's inequality $\operatorname{ratrk}(v)+$ $\operatorname{trdeg}_{X}(v) \leq \operatorname{dim}\left(O_{X, \xi}\right)$. Quasimonomial valuations are exactly the ones that give equality in the Abhyankar's inequality; see [JM12, Proposition 3.7].

Let $v \in \operatorname{Val}_{X}$ be a quasimonomial valuation. A $\log$ smooth pair $(Y, D)$ is said to be adapted to $v$ if $v \in \mathrm{QM}(Y, D)$. We say $(Y, D)$ is a good pair adapted to $v$ if $\left\{v\left(D_{i}\right) \mid v\left(D_{i}\right)>0\right\}$ are rationally independent.

Lemma 2.2 [JM12, Lemma 3.6]. Let $v \in \mathrm{Val}_{X}$ be a quasimonomial valuation. There exists a good pair $(Y, D)$ adapted to $v$. If $\left(Y^{\prime}, D^{\prime}\right) \succeq(Y, D)$ and $(Y, D)$ is a good pair adapted to $v$, then $\left(Y^{\prime}, D^{\prime}\right)$ is also a good pair adapted to $v$. 
An important class of valuations are divisorial valuations. A valuation is called divisorial if it is positively proportional to $\operatorname{ord}_{E}$ for some prime divisor $E$ over $X$, where $\operatorname{ord}_{E}$ is the vanishing order along $E$. One easily verifies that the trivial valuation is quasimonomial of rational rank zero, and a divisorial valuation is quasimonomial of rational rank one. Let $(Y, D)$ be a log smooth pair adapted to $v$. It can be verified that $v$ is divisorial if and only if $\mathbb{R}_{\geq 0}[v] \subseteq \mathrm{QM}_{\eta}(Y, D) \cong \mathbb{R}_{\geq 0}^{r}$ is a rational ray, that is, $\mathbb{R}_{\geq 0}[v]$ contains some rational point in $\mathbb{R}_{\geq 0}^{r}$.

For every $\log$ resolution $(Y, D)$ we can define the retraction map

$$
r_{Y, D}: \operatorname{Val}_{X} \rightarrow \mathrm{QM}(Y, D)
$$

by taking $v$ to a quasimonomial valuation in $\mathrm{QM}(Y, D)$ with $r_{Y, D}(v)\left(D_{i}\right)=v\left(D_{i}\right)$. Note that $r_{Y, D}$ is continuous and that $v \geq r_{Y, D}(v)$ with equality if and only if $v \in \mathrm{QM}(Y, D)$. Furthermore, if $\left(Y^{\prime}, D^{\prime}\right) \succeq(Y, D)$ is another resolution, then the retraction map $r_{Y, D}: \mathrm{QM}\left(Y^{\prime}, D^{\prime}\right) \rightarrow \mathrm{QM}(Y, D)$ (by abuse of notation if without confusion) is a surjective mapping that is integral linear on every $\mathrm{QM}_{\eta^{\prime}}\left(Y^{\prime}, D^{\prime}\right)$ and we have that $r_{Y, D} \circ r_{Y^{\prime}, D^{\prime}}=r_{Y, D}$. Therefore we can naturally regard $\mathrm{QM}(Y, D)$ as a subset of $\mathrm{QM}\left(Y^{\prime}, D^{\prime}\right)$, and hence of the set of quasimonomial valuations on $X$. Also note that $v(\mathfrak{a}) \geq r_{Y, D}(v)(\mathfrak{a})$ for an ideal $\mathfrak{a}$ on $X$, with equality if $(Y, D)$ is a $\log$ resolution of $\mathfrak{a}$; see [Lazarsfeld 2004] and [JM12, Corollary 4.8].

Tempered valuations. We first introduce the log discrepancy on an arbitrary scheme. Let $\pi: Y \rightarrow X$ be a birational proper morphism. The 0 -th Fitting ideal Fitt $0\left(\Omega_{Y / X}\right)$ is a locally principal ideal with its corresponding effective divisor denoted by $K_{Y / X}$; see [JM12, Section 1.3]. For a quasimonomial valuation $v \in \mathrm{QM}(Y, D)$, we define the log discrepancy

$$
A_{X}(v)=\sum v\left(D_{i}\right) \cdot A_{X}\left(\operatorname{ord}_{D_{i}}\right)=\sum v\left(D_{i}\right) \cdot\left(1+\operatorname{ord}_{D_{i}}\left(K_{Y / X}\right)\right) .
$$

We simply denote this by $A$ when the scheme $X$ is obvious. Note that $A$ is strictly positive linear on every $\mathrm{QM}_{\eta}(Y, D)$, and in particular continuous on every $\mathrm{QM}_{\eta}(Y, D)$ (or is weakly continuous according to Definition 3.4). Also note that if $\left(Y^{\prime}, D^{\prime}\right) \succeq(Y, D)$ and $v \in \mathrm{QM}\left(Y^{\prime}, D^{\prime}\right)$, then $A(v) \geq A\left(r_{Y, D}(v)\right)$ and equality holds only when $v \in \mathrm{QM}(Y, D)$. For an arbitrary valuation $v \in \operatorname{Val}_{X}$, we define

$$
A(v)=\sup _{(Y, D)} A\left(r_{Y, D}(v)\right) \in[0,+\infty] .
$$

Note that $A$ is lower-semicontinuous (lsc) as a valuative function.

Definition 2.3. A valuation $v$ is said to be tempered if $A(v)<\infty$. The valuation space $\mathrm{V}_{X}$ of $X$ is defined to be the space of tempered valuations as a subspace of $\operatorname{Val}_{X}$. 
We similarly denote by $\mathrm{V}_{X}^{*}$ the subset of nontrivial tempered valuations. If $f: X^{\prime} \rightarrow X$ is a proper birational morphism, then $A_{X}(v)=A_{X^{\prime}}(v)+v\left(K_{X^{\prime} / X}\right)$ (see [JM12, Proposition 5.1(3)]) and hence $\mathrm{V}_{X^{\prime}}=\mathrm{V}_{X}$. Since $\mathrm{V}_{X}$ is a topological subspace of $\mathrm{Val}_{X}$, it is naturally a subspace of the Berkovich space $X^{a n}$. See [JM12, Section 6.3] for a comparison.

With the aid of the log discrepancy, we can normalize $\mathrm{V}_{X}^{*}$ by letting $A(v)=1$, that is, we define $\Lambda_{X}:=\left\{v \in \mathrm{V}_{X}^{*} \mid A(v)=1\right\}$. In particular, we normalize every cone complex $\mathrm{QM}(Y, D)$ by setting $\Delta(Y, D):=\{v \in \mathrm{QM}(Y, D) \mid A(v)=1\}$. It is clear that $\Delta(Y, D)$ naturally possesses the structure of a simplicial complex, and by convention we say that $\Delta(Y, D)$ is a dual complex. Readers can compare the constructions here with [Boucksom et al. 2008; 2012b; 2012c].

The following lemma allows us to compare $v$ and ord $\mathrm{g}_{\xi}$, where $\xi=c_{X}(v)$, which is quite useful (see [Lazarsfeld 2004] or [JM12, Section 5.3] for the definition of $\operatorname{ord}_{\xi}$ ). See [JM12, Proposition 5.10] for a proof. Recently S. Boucksom, C. Favre and M. Jonsson [Boucksom et al. 2012a] gave a refinement of the following lemma.

Lemma 2.4 (Izumi-type inequality). Let $\xi=c_{X}(v)$ and $\mathfrak{m}_{\xi}$ be the defining ideal of $\overline{\{\xi\}}$. Then we have $v\left(\mathfrak{m}_{\xi}\right) \operatorname{ord}_{\xi} \leq v \leq A(v) \operatorname{ord}_{\xi}$.

Passing to the completion. A morphism $f: X^{\prime} \rightarrow X$ is regular if it is flat and its fibres are geometrically regular (see [JM12, Section 1.1]). The following lemma on $\log$ discrepancy is essential for finding a valuation that computes the log canonical threshold or norms in Section 5.

Lemma 2.5 [JM12, Proposition 5.13]. Let $f: X^{\prime} \rightarrow X$ be a regular morphism, and let $f_{*}: \mathrm{Val}_{X^{\prime}} \rightarrow \mathrm{Val}_{X}$ be the induced map. If $v^{\prime} \in \mathrm{Val}_{X^{\prime}}$ is a valuation on $X^{\prime}$, then $A\left(v^{\prime}\right) \geq A\left(f_{*}\left(v^{\prime}\right)\right)$. If we assume further that $X^{\prime}=\operatorname{Spec} \widehat{O_{X, \xi}}$ and $v^{\prime}$ is centred at the closed point of $X^{\prime}$, then $A\left(v^{\prime}\right)=A\left(f_{*}\left(v^{\prime}\right)\right)$.

Definition 2.6. If $\xi \in X$ is a point, then we define $\mathrm{V}_{X, \xi}:=c_{X}^{-1}(\xi)$ as a subspace of $\mathrm{V}_{X}$. We can normalize $\mathrm{V}_{X, \xi}$ by letting $v(\mathfrak{m})=1$, where $\mathfrak{m}$ is the defining ideal of $\overline{\{\xi\}}$. More precisely we define $\mathbb{V}_{X, \xi}:=\left\{v \in \mathrm{V}_{X, \xi} \mid v(\mathfrak{m})=1\right\}$. Let $M>0$ be a positive real number. We also define $\mathbb{V}_{X, \xi, M}:=\left\{v \in \mathbb{V}_{X, \xi} \mid A(v) \leq M\right\}$. According to [JM12, Proposition 5.9] the space $\mathbb{V}_{X, \xi, M}$ is compact. If $X=\operatorname{Spec} A$ and $\mathfrak{m}$ is the defining ideal of $\overline{\{\xi\}}$, we often use the notation $\mathbb{V}_{A, \mathfrak{m}}$ instead of $\mathbb{V}_{X, \xi}$.

Let $(R, \mathfrak{m})$ be a local ring. Given a tempered valuation $v \in \mathrm{V}_{R, \mathfrak{m}}$, we define $v^{\prime}(f)=\lim _{k \rightarrow \infty} v\left(\mathfrak{a}_{k}\right)$ for every $f \in \widehat{R}$, where $\mathfrak{a}_{k} \cdot \widehat{R}=f+\widehat{\mathfrak{m}}^{k}$. This is well-defined since $v\left(\mathfrak{a}_{k}\right) \leq A(v) \operatorname{ord}_{\xi}\left(\mathfrak{a}_{k}\right) \leq A(v) \operatorname{ord}_{\xi^{\prime}}(f)<\infty$ by Lemma 2.4. The above definition leads to a correspondence between the valuation spaces of Spec $R$ and Spec $\widehat{R}$ as follows. Throughout this paper we will use the notations $v$ and $v^{\prime}$ to indicate that $v=f_{*} v^{\prime}$ for simplicity if without confusion. 
Proposition 2.7. Let $(R, \mathfrak{m})$ be a local ring, and let $(\widehat{R}, \widehat{\mathfrak{m}})$ be its $\mathfrak{m}$-adic completion. If we denote by $f: \operatorname{Spec} \widehat{R} \rightarrow \operatorname{Spec} R$ the canonical morphism, then the induced map $f_{*}: \mathbb{V}_{\widehat{R}, \widehat{m}} \rightarrow \mathbb{V}_{R, \mathfrak{m}}$ is bijective. If $K^{\prime}$ is a compact subspace of $\mathbb{V}_{\widehat{R}, \widehat{\mathfrak{m}}}$, then $f_{*}$ is a homeomorphism from $K^{\prime}$ to its image. In particular, $\mathbb{V}_{\widehat{R}, \widehat{\mathfrak{m}}, M} \cong \mathbb{V}_{R, \mathfrak{m}, M}$ for any positive number $M$.

Proof. The bijectivity of $f_{*}$ follows from [JM12, Corollary 5.11], and we will prove the latter statement. Let $K=f_{*}\left(K^{\prime}\right)$. It suffices to show that $K^{\prime}$ is homeomorphic to $K$. Let $h \in \widehat{R}$ be a nonzero function. We have that $\max _{v^{\prime} \in K^{\prime}} v^{\prime}(h)=\alpha<\infty$ since $K^{\prime}$ is compact. If $g \in R$ is a nonzero function such that $g-h \in \widehat{\mathfrak{m}}^{n}$ in $\widehat{R}$ for some $n>\alpha$. Then $v^{\prime}(g-h) \geq n v^{\prime}(\widehat{\mathfrak{m}})>v^{\prime}(h)$ for all $v^{\prime} \in K^{\prime}$. It follows that $v(g)=v^{\prime}(g)=v^{\prime}(h)$ for all $v^{\prime} \in K^{\prime}$ and hence they induce the same topology.

\section{Functions on valuation spaces}

In this section we will discuss various classes of functions on valuation space with an emphasis on the quasi-plurisubharmonic (qpsh for short) functions.

Bounded homogeneous functions. Let $X$ be a scheme and $\mathrm{V}_{X}$ be its valuation space. A valuative function $\varphi$ is homogeneous if $\varphi(t v)=t \varphi(v)$ for all $v \in \mathrm{V}_{X}$ and $t \in \mathbb{R}_{+}$. A valuative function $\varphi$ is bounded if $\sup _{v \in \mathrm{V}_{X}^{*}}|\varphi(v)| / A(v)<\infty$. The set of bounded homogeneous functions (denoted by $\mathrm{BH}(X)$ ) forms an $\mathbb{R}$-linear space, which can be equipped with the norm $\|\varphi\|=\sup _{v \in \mathrm{V}_{X}^{*}}|\varphi(v)| / A(v)$. If $\mathfrak{q}$ is a nonzero ideal on $X$, then we define the $\mathfrak{q}$-norm as $\|\varphi\|_{\mathfrak{q}}=\sup _{v \in \mathrm{V}_{X}^{*}}|\varphi(v)| /(A(v)+v(\mathfrak{q}))$.

We also define

$$
\|\varphi\|_{\mathfrak{q}}^{+}:=\sup _{v \in \mathrm{V}_{X}^{*}} \frac{\varphi(v)}{A(v)+v(\mathfrak{q})}
$$

and

$$
\|\varphi\|_{\mathfrak{q}}^{-}:=\sup _{v \in \mathrm{V}_{X}^{*}} \frac{-\varphi(v)}{A(v)+v(\mathfrak{q})}
$$

Clearly, $\|\varphi\|_{\mathfrak{q}}^{+}=\|-\varphi\|_{\mathfrak{q}}^{-}$and $\|\cdot\|_{\mathfrak{q}}=\max \left\{\|\cdot\|_{\mathfrak{q}}^{+},\|\cdot\|_{\mathfrak{q}}^{-}\right\}$.

Lemma 3.1. Given two nonzero ideals $\mathfrak{p}, \mathfrak{q}$ on $X$, the $\mathfrak{p}$-norm and the $\mathfrak{q}$-norm are equivalent.

Proof. We first assume that $\mathfrak{p}=\mathrm{O}_{X}$. Then we have the inequalities

$$
\|\cdot\|_{\mathfrak{q}} \leq\|\cdot\| \leq\left(1+\sup _{v \in \mathrm{V}_{X}^{*}} \frac{v(\mathfrak{q})}{A(v)}\right)\|\cdot\|_{\mathfrak{q}} .
$$

Note that $\sup _{v \in \mathrm{V}_{X}^{*}} v(\mathfrak{q}) / A(v)=\max _{D_{i}}\left(\operatorname{ord}_{D_{i}}(\mathfrak{q}) / A\left(\operatorname{ord}_{D_{i}}\right)\right)<\infty$, where $D_{i}$ runs over all irreducible components of $D$ on a birational model $Y$ such that $(Y, D)$ is a $\log$ resolution of $\mathfrak{q}$. This implies that $1+\sup _{v \in \mathrm{V}_{X}^{*}} v(\mathfrak{q}) / A(v)<\infty$ and leads to the conclusion. 
Proposition 3.2. Given a scheme $X, \mathrm{BH}(X)$ is a Banach space. If $f: X^{\prime} \rightarrow X$ is a regular morphism and $f_{*}: \mathrm{V}_{X^{\prime}} \rightarrow \mathrm{V}_{X}$ is the induced map, then the induced map $f^{*}: \mathrm{BH}(X) \rightarrow \mathrm{BH}\left(X^{\prime}\right)$ by taking $\varphi$ to $\varphi \circ f_{*}$ is a bounded linear operator of Banach spaces. More precisely, $\left\|f^{*}(\varphi)\right\|_{\mathfrak{q} \cdot 0_{X^{\prime}}} \leq\|\varphi\|_{\mathfrak{q}}$ for any nonzero ideal $\mathfrak{q}$ on $X$.

Proof. Note that a bounded homogeneous function $\varphi$ is also a function on $\Lambda_{X}$, defined by $\left\{v \in \mathrm{V}_{X}^{*} \mid A(v)=1\right\}$, with the norm $\sup _{v \in \Lambda_{X}}|\varphi(v)|<\infty$. If $\left\{\varphi_{m}\right\}$ is a Cauchy sequence in $\mathrm{BH}(X)$, then $\varphi_{m}$ converges pointwise to a homogeneous function $\varphi$. Since $\sup _{v \in \Lambda_{X}}|\varphi(v)| \leq \sup _{v \in \Lambda_{X}}\left|\varphi(v)-\varphi_{m}(v)\right|+\sup _{v \in \Lambda_{X}}\left|\varphi_{m}(v)\right|<\infty$, $\varphi$ is a bounded homogeneous function. This proves that $\mathrm{BH}(X)$ is a Banach space. For the second statement, simply note that

$$
\left\|f^{*}(\varphi)\right\|_{\mathfrak{q} \cdot \bigcirc_{X^{\prime}}}=\sup _{v^{\prime} \in \mathrm{V}_{X^{\prime}}^{*}} \frac{|\varphi(v)|}{A\left(v^{\prime}\right)+v^{\prime}\left(\mathfrak{q} \cdot O_{X^{\prime}}\right)} \leq \sup _{v \in \mathrm{V}_{X}^{*}} \frac{|\varphi(v)|}{A(v)+v(\mathfrak{q})}=\|\varphi\|_{\mathfrak{q}} .
$$

by Lemma 2.5 .

Remark 3.3. Let $\varphi$ be a bounded homogeneous function such that $\varphi(v)=-v(\mathfrak{a})$ for some nonzero ideal $\mathfrak{a}$ on $X$. It is easy to see that the norm $\|\varphi\|_{\mathfrak{q}}$ is exactly the Arnold multiplicity $\operatorname{Arn}^{\mathfrak{q}} \mathfrak{a}$, and its reciprocal is the log canonical threshold $\operatorname{lct}^{\mathfrak{q}} \mathfrak{a}$. We will discuss this type of functions in detail later.

Definition 3.4. A bounded homogeneous function $\varphi$ is said to be weakly continuous if $\varphi$ is continuous on every dual complex $\Delta(Y, D)$.

Example 3.5. (1) As we already mentioned, the $\log$ discrepancy $A$ is a weakly bounded homogeneous function.

(2) If $\left\{\varphi_{k}\right\}$ is a sequence of continuous, bounded homogeneous functions that converges to a function $\varphi$ strongly in norm, then $\varphi$ is weakly continuous.

Ideal functions and qpsh functions. Given a nonzero ideal $\mathfrak{a}$, we define $|\mathfrak{a}|(v)=$ $-e^{v(\mathfrak{a})}$ by convention. It is obvious that $\log |\mathfrak{a}|$ is a continuous, bounded homogeneous function.

Definition 3.6. A bounded homogeneous function $\varphi$ is said to be an ideal function if there exists a finite number of nonzero ideals $\mathfrak{a}_{j}$ and positive real numbers $c_{j}$ such that $\varphi=\sum_{j=1}^{l} c_{j} \log \left|\mathfrak{a}_{j}\right|$.

Lemma 3.7. Let $\varphi=\sum_{j=1}^{l} c_{j} \log \left|\mathfrak{a}_{j}\right|$ be an ideal function on $X$ and $\mathfrak{q}$ be a nonzero ideal. Then,

$$
\|\varphi\|_{\mathfrak{q}}=\max _{E}\left\{\frac{\sum_{j=1}^{l} c_{j} \operatorname{ord}_{E} \mathfrak{a}_{j}}{A\left(\operatorname{ord}_{E}\right)+\operatorname{ord}_{E} \mathfrak{q}}\right\}
$$

for some prime divisor $E$ over $X$. 
Proof. Let $(Y, D)$ be a $\log$ resolution of $\mathfrak{q} \cdot \prod_{j=1}^{l} \mathfrak{a}_{j}$, and let $D_{i}$ 's be the irreducible components of $D$. By an easy computation, we see that

$$
\|\varphi\|_{\mathfrak{q}}=\max _{D_{i}}\left\{\frac{\sum_{j=1}^{l} c_{j} \operatorname{ord}_{D_{i}} \mathfrak{a}_{j}}{A\left(\operatorname{ord}_{D_{i}}\right)+\operatorname{ord}_{D_{i}} \mathfrak{q}}\right\},
$$

where $D_{i}$ runs over all irreducible components of $D$.

Lemma 3.8. Let $\varphi$ be a bounded homogeneous function which is determined on some dual complex $\Delta(Y, D)$ in the sense of $\varphi=\varphi \circ r_{Y, D}$. Assume that $\varphi$ is affine on each face of the dual complex $\Delta(Y, D)$ and that $\left(Y^{\prime}, D^{\prime}\right) \succeq(Y, D)$. Then $\varphi=\varphi \circ r_{Y^{\prime}, D^{\prime}}$ which is also affine on each face of the dual complex $\Delta\left(Y^{\prime}, D^{\prime}\right)$.

Proof. The assumption that $\varphi$ is affine on each face of the dual complex $\Delta(Y, D)$ is equivalent to that $\varphi$ is linear on each simplicial cone of $\mathrm{QM}(Y, D)$. The conclusion follows from the fact that $r_{Y, D}$ is linear on each simplicial cone of $\mathrm{QM}\left(Y^{\prime}, D^{\prime}\right)$.

Definition 3.9. A bounded homogeneous function $\varphi$ is a quasi-plurisubharmonic (qpsh for short) function if there exists a sequence of ideal functions that converges to $\varphi$ strongly in norm. The set of qpsh functions, which is a closed convex cone in $\mathrm{BH}(X)$, is denoted by $\mathrm{QPSH}(X)$.

Definition 3.10. The support of a qpsh function is the set of elements of the form $c_{X}(v)$, for some nontrivial tempered valuation $v$ such that $\varphi(v)<0$.

If $\varphi=\sum_{j=1}^{l} c_{i} \log \left|\mathfrak{a}_{i}\right|$ is an ideal function, then the support of $\varphi$ is the union of the vanishing loci $V\left(\mathfrak{a}_{j}\right)$ and hence proper closed. We will see that the support of a qpsh function is a countable union of proper closed subsets. See Corollary 4.26.

Proposition 3.11. Let $\varphi \in \mathrm{QPSH}(X)$ be a qpsh function. Then, $\varphi$ is convex on each face of every dual complex $\Delta(Y, D)$. Moreover, $\varphi \circ r_{Y, D}$ form a decreasing net of continuous functions that converges to $\varphi$ strongly in norm. In particular, $\varphi$ is weakly continuous and upper-semicontinuous (usc for short).

Proof. To show that $\varphi$ is convex on each face of every dual complex $\Delta(Y, D)$, it suffices to prove this when $\varphi$ is an ideal function. We can assume that $\varphi=c \log |\mathfrak{a}|$. Let $\eta$ be a generic point of the intersection of $D_{1}, \ldots, D_{l}$. We will prove that $\varphi$ is convex on $\mathrm{QM}_{\eta}(Y, D)$, which essentially implies the convexity on $\Delta(Y, D)$. To this end, assume that $v=\sum_{j=1}^{k} \lambda_{j} v_{j}$ such that $v, v_{j} \in \mathrm{QM}_{\eta}(Y, D), \lambda_{j}>0$ for every $j$ and $\sum_{j=1}^{k} \lambda_{j}=1$. Assume further that $\mathfrak{a} \cdot O_{Y}$ is principal near $\eta$ generated by $f$. If we consider the local coordinates $y=\left\{y_{1}, \ldots, y_{l}\right\}$ with the origin $\eta$, then $v$ and $v_{j}$ can be represented by $\alpha=\left(\alpha^{1}, \ldots, \alpha^{l}\right)$ and $\alpha_{j}=\left(\alpha_{j}^{1}, \ldots, \alpha_{j}^{l}\right)$ with the values $v(f)=\min \left\{\langle\alpha, \beta\rangle \mid f=\sum c_{\beta} y^{\beta}\right\}$ and $v_{j}(f)=\min \left\{\left\langle\alpha_{j}, \beta\right\rangle \mid f=\sum c_{\beta} y^{\beta}\right\}$. Obviously, $v(f) \geq \sum_{j=1}^{k} \lambda_{j} v_{j}(f)$ and we obtain the required convexity. If $\mathfrak{a} \cdot \mathscr{O}_{Y}$ is not principal, then $\varphi$ is the maximum of a finite number of convex functions and hence convex. 
Given an arbitrary qpsh function $\varphi$, the functions $\varphi \circ r_{Y, D}$ form a decreasing net because $v \leq r_{Y, D}(v)$, and $\varphi$ is continuous on $\Delta(Y, D)$ because it is the uniform limit function of continuous functions. It suffices to show that $\varphi \circ r_{Y, D}$ converges to $\varphi$ strongly in norm. To this end, consider a sequence of ideal functions $\varphi_{j}=c_{j} \log \left|\mathfrak{a}_{j}\right|$ that converges to $\varphi$ strongly in norm. We then obtain that

$$
\left\|\varphi-\varphi \circ r_{Y, D}\right\| \leq\left\|\varphi-\varphi_{j}\right\|+\left\|\varphi_{j}-\varphi \circ r_{Y, D}\right\| \leq 2\left\|\varphi-\varphi_{j}\right\|
$$

if $(Y, D)$ is a $\log$ resolution of $\mathfrak{a}_{j}$, which completes the proof.

Remark 3.12. The proposition above implies that a qpsh function is uniquely determined by its values on divisorial valuations. In fact, if $\varphi$ and $\phi$ have the same values on divisorial valuations, then $\varphi=\phi$ on every dual complex $\Delta(Y, D)$ by continuity and hence $\varphi=\phi$ on $\mathrm{V}_{X}$.

The following example shows that the pointwise limit of a decreasing sequence of ideal functions is not qpsh in general.

Example 3.13. Let $X=\operatorname{Spec} k[x]$ be an affine line, and let $\phi_{k}=\sum_{j=1}^{k} \log \left|f_{j}\right|$, where $f_{j}=x-j$. We see that $\phi_{k}$ is a decreasing sequence of ideal functions and the pointwise limit function $\varphi$ exists. But $\varphi$ is not qpsh because $\|\varphi-\phi\| \geq 1$ for any ideal function $\phi$.

If $f: X^{\prime} \rightarrow X$ is a regular morphism and $\varphi$ is a qpsh function on $X$, then $f^{*} \varphi$ is a qpsh function on $\mathrm{V}_{X^{\prime}}$ by Proposition 3.2. In particular if $f: U \rightarrow X$ (resp. $f: \operatorname{Spec} \mathrm{O}_{X, \xi} \rightarrow X$ ) is an open inclusion, we say that $f^{*} \varphi$ is the restriction (resp. germ) of $\varphi$, denoted by $\left.\varphi\right|_{U}$ (resp. $\varphi_{\xi}$ ). Also, restrictions to the neighbourhoods of a point $\xi$ induce a map $\operatorname{QPSH}(X) \rightarrow \lim _{U \ni \xi} \operatorname{QPSH}(U)$, and the image of $\varphi$ is also said to be the germ of $\varphi$, denoted by $\vec{\varphi}_{\xi}$.

If $\xi$ is not contained in the support of a qpsh function $\varphi$, then $\varphi_{\xi}=0$ by Proposition 3.11. However, the following example shows that it could happen that the germ of $\varphi$ is nonzero in the set $\lim _{U \ni \xi} \operatorname{QPSH}(U)$.

Example 3.14. Let $X=\operatorname{Spec} k[x]$ be an affine line, and let $\phi_{k}=\sum_{m=1}^{k} 2^{-m} \log \left|f_{m}\right|$, where $f_{m}=x-1 / m$. It is easy to see that $\phi_{k}$ converges to a function $\phi$ strongly in norm. Note that the origin is not contained in the support of $\phi$, but the germ of $\phi$ in $\lim _{U \ni 0} \operatorname{QPSH}(U)$ is nonzero.

From this example we see that if we define $\left\|\left.\varphi\right|_{\xi}\right\|:=\inf _{U \ni \xi}\left\|\left.\varphi\right|_{U}\right\|$, then $\|\cdot\|$ is only a seminorm.

Proposition 3.15. There is a surjective map of convex cones

$$
r: \underset{U \ni \xi}{\lim _{U \xi}} \operatorname{QPSH}(U) \rightarrow \operatorname{QPSH}\left(\operatorname{Spec} \mathrm{O}_{X, \xi}\right)
$$

which preserves the seminorm, and also preserves $\|\cdot\|^{+}$and $\|\cdot\|^{-}$. 
Proof. If $\varphi=c \log |\mathfrak{a}|$ and $\varphi^{\prime}=c^{\prime} \log |\mathfrak{a}|$, then we claim that $\left\|\left.\varphi\right|_{\xi}-\left.\varphi^{\prime}\right|_{\xi}\right\|=\left\|\varphi_{\xi}-\varphi_{\xi}^{\prime}\right\|$. To this end, let $\mu:(Y, D) \rightarrow X$ be a log resolution of $\mathfrak{a} \cdot \mathfrak{a}^{\prime}$, and let $\mathfrak{a} \cdot \mathscr{O}_{Y}=\mathscr{O}_{Y}(-F)$ and $\mathfrak{a}^{\prime} \cdot \mathrm{O}_{Y}=\mathrm{O}_{Y}\left(-F^{\prime}\right)$. One can easily check that

$$
\left\|\left.\varphi\right|_{\xi}-\left.\varphi^{\prime}\right|_{\xi}\right\|=\max _{D_{i} \in \mathscr{S}} \frac{\left|\operatorname{ord}_{D_{i}} F-\operatorname{ord}_{D_{i}} F^{\prime}\right|}{A\left(\operatorname{ord}_{D_{i}}\right)},
$$

where $\mathscr{Y}$ consists of irreducible components $D_{i}$ of $D$ such that $\mu\left(D_{i}\right)$ contains $\xi$ in its support. This implies the claim.

Given a qpsh function $\varphi_{\xi} \in \operatorname{QPSH}\left(\operatorname{Spec} 0_{X, \xi}\right)$, there exists a sequence of ideal functions $\varphi_{\xi, i}=c_{i} \log \left|\mathfrak{a}_{\xi, i}\right|$ that converges to $\varphi_{\xi}$ strongly in norm. Let $\mathfrak{a}_{i}$ be ideals on $X$ such that $\mathfrak{a}_{i} \cdot \mathscr{O}_{X, \xi}=\mathfrak{a}_{\xi, i}$. We have that $\varphi_{i}=c_{i} \log \left|\mathfrak{a}_{i}\right|$ converges to a qpsh function in $\lim _{U \ni \xi} \operatorname{QPSH}(U)$ strongly in norm due to the previous claim. Therefore we obtain the surjectivity of $r$.

Finally, for two qpsh functions $\varphi$ and $\varphi^{\prime}$ on an open neighbourhood of $\xi$, the equality $\left\|\left.\varphi\right|_{\xi}-\left.\varphi^{\prime}\right|_{\xi}\right\|=\left\|\varphi_{\xi}-\varphi_{\xi}^{\prime}\right\|$ follows from the claim in the first paragraph. Apply a similar argument to $\|\cdot\|^{+}$and $\|\cdot\|^{-}$, we obtain the conclusion.

From the discussion above, we see that $\left.\varphi\right|_{\xi}$ provides more information while it is not a valuative function. We sometimes identify $\left.\varphi\right|_{\xi}$ and $\varphi_{\xi}$ as the germ of $\varphi$ at $\xi$.

\section{Multiplier ideals}

We will now discuss the multiplier ideals of qpsh functions. Recall that a graded sequence of ideals $\mathfrak{a}_{\bullet}$ is a sequence of ideals that satisfies $\mathfrak{a}_{m} \cdot \mathfrak{a}_{n} \subseteq \mathfrak{a}_{m+n}$. By convention we put $\mathfrak{a}_{0}=\mathscr{O}_{X}$, and we say $\mathfrak{a}_{\bullet}$ is nontrivial if $\mathfrak{a}_{m} \neq 0$ for some positive integer $m$. Note that in this case there are infinitely many $m$ such that $\mathfrak{a}_{m} \neq 0$. A subadditive sequence of ideals $\mathfrak{b}_{\bullet}$ is a one-parameter family $\mathfrak{b}_{t}$ satisfying $\mathfrak{b}_{s} \cdot \mathfrak{b}_{t} \supseteq \mathfrak{b}_{s+t}$ for every $s, t \in \mathbb{R}_{+}$. Similarly, we put $\mathfrak{b}_{0}=\mathrm{O}_{X}$ and we say that $\mathfrak{b}_{\bullet}$ is nontrivial if $\mathfrak{b}_{t} \neq 0$ for all $t \in \mathbb{R}_{+}$. Throughout this paper, every sequence of ideals is assumed to be nontrivial. We define $v\left(\mathfrak{a}_{\bullet}\right)=\inf _{m \geq 1} v\left(\mathfrak{a}_{m}\right) / m$ and $v\left(\mathfrak{b}_{\bullet}\right)=\sup _{t>0} v\left(\mathfrak{b}_{t}\right) / t$ as in [Ein et al. 2006]. We similarly define $\left|\mathfrak{a}_{\bullet}\right|(v)=e^{-v\left(\mathfrak{a}_{\bullet}\right)}$ and $\left|\mathfrak{b}_{\bullet}\right|(v)=e^{-v\left(\mathfrak{b}_{\bullet}\right)}$ for a graded sequence and a subadditive sequence of ideals respectively.

\section{Multiplier ideals.}

Definition 4.1. For a bounded homogeneous function $\varphi \in \mathrm{BH}(X)$, the multiplier ideal $\mathscr{g}(\varphi)$ of $\varphi$ is the largest ideal in the set of nonzero ideals $\left\{\mathfrak{a}\left|\|\log |\mathfrak{a}|-\varphi\|^{+}<1\right\}\right.$. If this set is empty, then we define $\mathscr{f}(\varphi)=(0)$.

Remark 4.2. We will see that the set above is always nonempty when $\varphi$ is qpsh and $\mathscr{f}(\varphi)$ is therefore nonzero (see Remark 4.21). Moreover, we have the inequality $\varphi \leq \log |\mathscr{f}(\varphi)|$ (see Remark 4.21), and hence $\|\log |\mathscr{F}(\varphi)|-\varphi\|<1$ holds. 
The following proposition shows that our definition of multiplier ideals coincides with the classical definition of multiplier ideals.

Proposition 4.3. If $\varphi$ is an ideal function and we write $\varphi=\sum_{i=1}^{l} c_{i} \log \left|\mathfrak{a}_{i}\right|$, then $\mathscr{F}(\varphi)=\mathscr{S}\left(\prod_{i=1}^{l} \mathfrak{a}_{\mathfrak{i}}{ }^{c_{i}}\right)$.

Proof. Let $\pi:(Y, D) \rightarrow X$ be a $\log$ resolution of $\prod_{i=1}^{l} \mathfrak{a}_{\mathfrak{i}}$, and $\mathfrak{a}_{i} \cdot \mathscr{O}_{Y}=\mathscr{O}_{Y}\left(-F_{i}\right)$ with $F_{i}$ being supported in $D$. Since $\mathscr{E}\left(\prod_{i=1}^{l} \mathfrak{a}_{\mathfrak{i}}{ }^{c_{i}}\right)=\pi_{*} \hat{O}_{Y}\left(K_{Y / X}-\left\llcorner\sum_{i=1}^{l} c_{i} F_{i}\right\lrcorner\right)$, it is easy to check that $\left\|\log \left|\mathscr{F}\left(\prod_{i=1}^{l} \mathfrak{a}_{i}^{c_{i}}\right)\right|-\varphi\right\|^{+}<1$, which immediately implies that $\mathscr{f}(\varphi) \supseteq \mathscr{g}\left(\prod_{i=1}^{l} \mathfrak{a}_{\mathfrak{i}}{ }^{c_{i}}\right)$.

Conversely, if $f \in \Gamma(U, \mathscr{f}(\varphi))$ is a regular function on an affine open subset $U$, then $\left\|\log |f|-\left.\varphi\right|_{U}\right\|^{+}<1$. It follows that $v(f)+A(v)+\varphi(f)>0$ for all nontrivial tempered valuations $v$ on $U$. In particular, $\operatorname{ord}_{E} f+\operatorname{ord}_{E} K_{Y / X}+1>-\varphi\left(\operatorname{ord}_{E}\right)$ for any prime divisor $E$ on $\pi^{-1} U$. Thus $f \in \Gamma\left(U, \mathscr{f}\left(\prod_{i=1}^{l} \mathfrak{a}_{i}{ }^{c_{i}}\right)\right)$ and it follows that $\mathscr{f}(\varphi) \subseteq \mathscr{E}\left(\prod_{i=1}^{l} \mathfrak{a}_{\mathfrak{i}}{ }^{c_{i}}\right)$.

The lemmas below will be frequently used in this paper.

Lemma 4.4. Given a nonzero ideal $\mathfrak{q}$ and a qpsh function $\varphi \in \operatorname{QPSH}(X), \mathfrak{q} \subseteq \Phi(\lambda \varphi)$ if and only if $\lambda^{-1}>\|\varphi\|_{\mathfrak{q}}$. Thus $\|\varphi\|_{\mathfrak{q}}^{-1}=\min \{t \mid \mathfrak{q} \nsubseteq \mathscr{g}(t \varphi)\}$.

Proof. If $\mathfrak{q} \subseteq \mathscr{g}(\lambda \varphi)$, then $\|\log |\mathfrak{q}|-\lambda \varphi\|^{+}<1$ by definition. That is,

$$
\sup _{v \in \mathrm{V}_{X}^{*}} \frac{-v(\mathfrak{q})-\lambda \varphi(v)}{A(v)}<1 .
$$

This implies that $-v(\mathfrak{q})-\lambda \varphi(v) \leq(1-\varepsilon) A(v)$ for every $v \in \mathrm{V}_{X}^{*}$. Thus

$$
\frac{-\lambda \varphi(v)}{A(v)+v(\mathfrak{q})} \leq \frac{(1-\varepsilon) A(v)+v(\mathfrak{q})}{A(v)+v(\mathfrak{q})} \leq(1-\varepsilon)+\varepsilon\|\log |\mathfrak{q}|\|_{\mathfrak{q}}<1
$$

by Lemma 3.7. We obtain $\lambda^{-1}>\|\varphi\|_{\mathfrak{q}}$ by definition.

Conversely we assume that $\|\varphi\|_{\mathfrak{q}}=\sup _{v \in \mathrm{V}_{X}^{*}}(-\lambda \varphi(v)) /(A(v)+v(\mathfrak{q}))<1$. Then $-\lambda \varphi(v) \leq(1-\varepsilon)(A(v)+v(\mathfrak{q}))$. Therefore

$$
\frac{-v(\mathfrak{q})-\lambda \varphi(v)}{A(v)} \leq 1-\varepsilon-\varepsilon \frac{v(\mathfrak{q})}{A(v)} \leq 1-\varepsilon
$$

for a sufficiently small $\varepsilon$ which leads to the conclusion $\mathfrak{q} \subseteq \mathscr{f}(\lambda \varphi)$.

Lemma 4.5. Let $\xi$ be a point of a scheme $X$, and $\varphi$ be a qpsh function. Assume that the multiplier ideal $\mathscr{F}(\varphi)$ is nonzero. (In fact, this assumption automatically holds by Lemma 4.20 and Remark 4.21.) Then:

(1) $\mathscr{J}\left(\left.\varphi\right|_{U}\right)=\mathscr{F}(\varphi) \cdot \mathrm{O}_{U}$.

(2) $\mathscr{F}\left(\varphi_{\xi}\right)=\mathscr{F}(\varphi) \cdot O_{X, \xi}$.

(3) $\operatorname{Set} \lambda^{-1}:=\|\varphi\|_{\mathfrak{q}}$. If $\xi \in \mathrm{V}(\mathscr{g}(\lambda \varphi): \mathfrak{q})$, then $\|\varphi\|_{\mathfrak{q}}=\left\|\varphi_{\xi}\right\|_{\mathfrak{q} \cdot \odot_{X, \xi}}$. 
Proof. (1) Since $\left\|\log \left|\mathscr{g}(\varphi) \cdot \mathscr{O}_{U}\right|-\left.\varphi\right|_{U}\right\|^{+} \leq\|\log |\mathscr{g}(\varphi)|-\varphi\|^{+}<1$, we have $\mathscr{F}(\varphi) \cdot \mathfrak{O}_{U} \subseteq \mathscr{g}\left(\left.\varphi\right|_{U}\right)$. On the other hand, if we denote by $\mathfrak{m}$ the defining ideal of $X \backslash U$, then there exists a sufficiently large integer $k$ such that $v(\mathscr{g}(\varphi)) \leq v\left(\mathfrak{m}^{k}\right)$ for all valuations $v$ centred in $X \backslash U$. Now we extend $\mathscr{E}\left(\left.\varphi\right|_{U}\right)$ to $X$ and still denote it by $\mathscr{F}\left(\left.\varphi\right|_{U}\right)$. Therefore $\left\|\log \left|\mathscr{F}\left(\left.\varphi\right|_{U}\right) \cdot \mathfrak{m}^{k}\right|-\varphi\right\|^{+}<1$, which implies $\mathscr{g}\left(\left.\varphi\right|_{U}\right) \subseteq \mathscr{g}(\varphi) \cdot \mathfrak{O}_{U}$.

(2) First note that $\left\|\log \left|\mathscr{}(\varphi) \cdot O_{X, \xi}\right|-\varphi_{\xi}\right\|^{+} \leq\|\log |\mathscr{f}(\varphi)|-\varphi\|^{+}<1$, and it follows that $\mathscr{F}(\varphi) \cdot \mathscr{O}_{X, \xi} \subseteq \mathscr{F}\left(\varphi_{\xi}\right)$. For the inverse inclusion, we see that if $f \in \mathscr{F}\left(\varphi_{\xi}\right)$, then there exists an open neighbourhood $U$ of $\xi$ such that $\left\|\log |f|-\left.\varphi\right|_{U}\right\|^{+}<1$ by Proposition 3.15. Thus $f \in \mathscr{E}\left(\left.\varphi\right|_{U}\right) \cdot \mathscr{O}_{X, \xi}=\mathscr{F}(\varphi) \cdot \mathscr{O}_{X, \xi}$.

(3) It is obvious that $\|\varphi\|_{\mathfrak{q}} \geq\left\|\varphi_{\xi}\right\|_{\mathfrak{q} \cdot 0_{X, \xi}}$ by Proposition 3.2. If $\xi \in \mathrm{V}(\mathscr{g}(\lambda \varphi): \mathfrak{q})$, then $\left(\mathscr{F}\left(\lambda \varphi_{\xi}\right): \mathfrak{q} \cdot \mathscr{O}_{X, \xi}\right)=(\mathscr{E}(\lambda \varphi): \mathfrak{q}) \cdot \mathscr{O}_{X, \xi} \neq 0_{X, \xi}$. Therefore $\mathfrak{q} \cdot \mathscr{O}_{X, \xi} \nsubseteq \mathscr{F}\left(\left.\lambda \varphi\right|_{\xi}\right)$ and $\lambda^{-1} \leq\left\|\varphi_{\xi}\right\|_{\mathfrak{q} \cdot \mathscr{O}_{X, \xi}}$ by Lemma 4.4 .

\section{Algebraic qpsh functions.}

Definition 4.6. A qpsh function $\varphi \in \operatorname{QPSH}(X)$ is algebraic if it is the limit function of an increasing sequence of ideal functions $\varphi=\lim _{m \rightarrow \infty} \varphi_{m}$ (in norm). Note that $\varphi$ being algebraic implies that $t \varphi$ is algebraic for any $t \in \mathbb{R}_{>0}$, and that $\varphi+\psi$ is algebraic provided that $\psi$ is another algebraic qpsh function. Thus the set of algebraic qpsh functions is a convex subcone of $\mathrm{QPSH}(X)$, denoted by $\mathrm{QPSH}^{a}(X)$.

An algebraic function is lower-semicontinuous (lsc for short) on $\mathrm{V}_{X}$ by its definition, and usc by Proposition 3.11, so it is continuous. We will see that in Definition 4.6 the phrase "in norm" is not necessary; that is, the pointwise limit of an increasing sequence of ideal functions is algebraic qpsh (see Lemma 4.15). One can compare this fact with Remark 4.25. The following example shows that a qpsh function is not necessarily algebraic.

Example 4.7. Let $X=\operatorname{Spec} k\left[x_{1}, x_{2}\right]$ be the affine plane. If we set

$$
\phi_{k}=\sum_{l=1}^{k} \frac{1}{2^{l}} \log \left|f_{l}\right|, \quad \text { where } f_{l}=x_{1}+x_{2}^{2^{l}},
$$

then $\phi_{k}$ converges to a qpsh function $\phi$ strongly in norm. However, the qpsh function $\phi$ is not algebraic since there is no ideal function $\varphi \leq \phi$.

The following lemma shows that a graded system of ideals naturally induces an algebraic qpsh function.

Lemma 4.8. Let $\mathfrak{a}_{\bullet}$ be a graded sequence of ideals. If we let $\log \left|\mathfrak{a}_{\bullet}\right|(v)=v\left(\mathfrak{a}_{\bullet}\right)$, then $\log \left|\mathfrak{a}_{\mathbf{0}}\right|$ is an algebraic qpsh function.

Proof. It suffices to find a subsequence of $\left\{\mathfrak{a}_{m}\right\}$ such that $\left\{\varphi_{k}:=\left(1 / m_{k}\right) \log \left|\mathfrak{a}_{m_{k}}\right|\right\}$ is an increasing sequence of ideal functions that converges to a qpsh function strongly in norm. Let $\mathfrak{b}_{\bullet}$ be a sequence of ideals such that $\mathfrak{b}_{t}=\mathscr{g}\left(\mathfrak{a}_{\bullet}^{t}\right)$ (see [Lazarsfeld 
2004]). Note that $\mathfrak{b}_{\text {• }}$ is subadditive of controlled growth (see [JM12, Section 2, Section 6, Appendix]). Now we fix an integer $m$ such that $\mathfrak{a}_{m} \neq 0$. Since $\mathfrak{b}_{m} \supseteq$ $\mathscr{f}\left(\mathfrak{a}_{m}\right) \supseteq \mathfrak{a}_{m}$, we have $v\left(\mathfrak{b}_{m}\right) \leq v\left(\mathfrak{a}_{m}\right)$. Since $v\left(\mathfrak{b}_{m}\right)+A(v)-(1 / k) v\left(\mathfrak{a}_{m k}\right)>0$ for all nontrivial tempered valuations $v$, where $k$ is a sufficiently divisible integer, we have $v\left(\mathfrak{a}_{m k}\right) /(m k)<v\left(\mathfrak{b}_{m}\right) / m+A(v) / m$. From the inequalities

$$
\frac{v\left(\mathfrak{b}_{m}\right)}{m} \leq \frac{v\left(\mathfrak{a}_{m k}\right)}{m k}<\frac{v\left(\mathfrak{b}_{m}\right)}{m}+\frac{A(v)}{m}
$$

we have $\left\|\frac{1}{m k} \log \left|\mathfrak{a}_{m k}\right|-\frac{1}{m k l} \log \left|\mathfrak{a}_{m k l}\right|\right\|<\frac{1}{m}$ for every positive integer $l$. As we multiply $m$, we obtain the desired sequence of ideal functions.

Definition 4.9. Let $\varphi \in \mathrm{BH}(X)$ be a bounded homogeneous function. Its envelope ideal $\mathfrak{a}(\varphi)$ is the largest ideal in the set $\{\mathfrak{a}|\log | \mathfrak{a} \mid \leq \varphi\}$ if this set is nonempty. If it is empty, we set $\mathfrak{a}(\varphi)=0$.

Proposition 4.10. If $\varphi$ is qpsh and $\mathfrak{a}(\varphi)$ is nonzero, then the envelope ideal can be written explicitly as $\Gamma(U, \mathfrak{a}(\varphi)):=\left\{f \in \mathcal{O}_{X}(U) \mid v(f)+\varphi(v) \geq 0\right.$ for every $\left.v \in \mathrm{V}_{U}^{*}\right\}$ on every open subset $U$.

Proof. Since the question is local, we can assume that $X=\operatorname{Spec} A$ is affine. It suffices to prove that the ideal $\mathfrak{a}$, defined by

$$
\mathfrak{a}(U):=\left\{f \in \mathcal{O}_{X}(U) \mid v(f)+\varphi(v) \geq 0 \text { for every } v \in \mathrm{V}_{U}^{*}\right\}
$$

on every open subset $U$, is coherent. To this end, we write $I:=\mathfrak{a}(X)$, and we will prove that $\mathfrak{a}\left(U_{g}\right)=I_{g}$ for any nonzero regular function $g \in A$, where $U_{g}$ denotes the affine open subset defined by $g$. Since $\mathfrak{a}\left(U_{g}\right) \supseteq I_{g}$ by definition, we only need to prove the converse inclusion. Note that there exists a large integer $k$ such that $k v(g) \geq v(\mathfrak{a}(\varphi))$ for every nontrivial tempered valuation $v$ centred in the locus $V(g)$, and hence $k \log |g|(v) \leq \varphi(v)$. If $f$ is a regular function on $U_{g}$ such that $v(f)+\varphi(v) \geq 0$ for every $v \in \mathrm{V}_{U_{g}}^{*}$, then $v\left(f g^{k}\right)+\varphi(v) \geq 0$ for every $v \in \mathrm{V}_{X}^{*}$. This implies that $f \in I_{g}$.

If we set $\mathfrak{a}(\varphi)_{m}=\mathfrak{a}(m \varphi)$, then $\left\{\mathfrak{a}(\varphi)_{\bullet}\right\}$ is a (possibly trivial) graded sequence of ideals. The following lemma shows that every algebraic qpsh function is of the form $\log \left|\mathfrak{a}_{\bullet}\right|$.

Lemma 4.11. If $\varphi \in \operatorname{QPSH}^{a}(X)$ is an algebraic qpsh function, then $\varphi=\log \mid \mathfrak{a}(\varphi)$ •|.

Proof. Given an arbitrarily small positive number $\varepsilon$, there exist an ideal $\mathfrak{a}$ on $X$ and an integer $m$ such that $\frac{1}{m} \log |\mathfrak{a}| \leq \varphi$ and $\left\|\frac{1}{m} \log |\mathfrak{a}|-\varphi\right\|<\varepsilon$. We have $\frac{1}{m} \log \left|\mathfrak{a}(\varphi)_{m}\right| \geq \frac{1}{m} \log |\mathfrak{a}|$ by definition and the conclusion follows.

By combining Proposition 4.3, Lemmas 4.8 and 4.11, we see that a bounded homogeneous function is algebraic qpsh if and only if it is derived from a graded sequence of ideals. Readers can compare the following theorem with Theorem 4.24. 
Theorem 4.12. If $\varphi$ is a bounded homogeneous function, the following statements are equivalent.

(1) $\varphi \in \mathrm{QPSH}^{a}(X)$ is algebraic qpsh.

(2) There exists a graded sequence of ideals $\mathfrak{a}_{\bullet}$ such that $\varphi=\log \left|\mathfrak{a}_{\bullet}\right|$.

(3) The graded system of ideals $\mathfrak{a}(\varphi)$ • is nontrivial and $\varphi=\log |\mathfrak{a}(\varphi) \bullet|$.

Proof. If we assume (1), then (3) holds by Lemma 4.11. Note that (3) implies (2) if we simply put $\mathfrak{a}_{\bullet}=\mathfrak{a}(\varphi)$. Finally, (1) follows from (2) by Lemma 4.8.

We will use the following easy lemma. For the convenience of readers we present a proof here.

Lemma 4.13. Let $\varphi \in \mathrm{QPSH}^{a}(X)$ be an algebraic qpsh function.

(1) Assume that $\left\{\varphi_{m}\right\}$ is an increasing sequence of qpsh functions that converges to $\varphi$ strongly in norm. Then $\mathscr{F}(\varphi)=\mathscr{F}\left(\varphi_{m}\right)$ for sufficiently large $m$.

(2) Assume that $f: X^{\prime} \rightarrow X$ is a regular morphism of schemes. Then $f^{*} \varphi$ is algebraic qpsh.

Proof. (1) We see that $\|\log |\mathscr{\mathscr { S }}(\varphi)|-\varphi\|^{+}=1-\varepsilon$ for some positive number $\varepsilon$. If $\left\|\varphi-\varphi_{m}\right\|<\varepsilon$, then $\left\|\log |\mathscr{f}(\varphi)|-\varphi_{m}\right\|^{+}<1$ and $\mathscr{f}(\varphi) \subseteq \mathscr{F}\left(\varphi_{m}\right)$. The inverse inclusion $\mathscr{F}(\varphi) \supseteq \mathscr{F}\left(\varphi_{m}\right)$ is obvious because $\varphi \geq \varphi_{m}$.

(2) Assume $\varphi_{m}$ is an increasing sequence of ideal functions that converges to $\varphi$ strongly in norm. Then $f^{*} \varphi_{m}$ is also an increasing sequence of ideal functions that converges to $f^{*} \varphi$ strongly in norm by Proposition 3.2. This implies that $f^{*} \varphi$ is algebraic qpsh.

By combining Lemmas 4.8 and 4.13(1), we see that the definition of valuative multiplier ideals of algebraic functions coincides with the classical definition of asymptotic multiplier ideals.

Corollary 4.14. Let $\mathfrak{a}_{\bullet}$ be a graded sequence of ideals. If we write $\varphi=\log \left|\mathfrak{a}_{\bullet}\right|$, then $\mathscr{g}(\varphi)=\mathscr{g}\left(\mathfrak{a}_{\bullet}\right)$.

\section{General qpsh functions.}

Lemma 4.15. If $\left\{\varphi_{\lambda}\right\}$ is a family of (algebraic) qpsh functions, then $\sup _{\lambda} \varphi_{\lambda}$ is an (algebraic) qpsh function. Therefore, the convex cone $\mathrm{QPSH}(X)\left(\right.$ resp. $\left.\mathrm{QPSH}^{a}(X)\right)$ is closed under taking the supremum.

Proof. We firstly assume that $\left\{\varphi_{\lambda}\right\}$ is a family of algebraic qpsh functions, and we write $\psi=\sup _{\lambda} \varphi_{\lambda}$. Since $\psi \geq \varphi_{\lambda}$ for every $\lambda, \mathfrak{a}(\psi)_{m} \supseteq \mathfrak{a}\left(\varphi_{\lambda}\right)_{m}$. It follows that $\log \left|\mathfrak{a}(\psi)_{\bullet}\right| \geq \log \left|\mathfrak{a}\left(\varphi_{\lambda}\right)_{\bullet}\right|=\varphi_{\lambda}$. Therefore $\psi=\log \left|\mathfrak{a}(\psi)_{\bullet}\right|$ is algebraic qpsh.

Now we treat the case when $\left\{\varphi_{\lambda}\right\}$ is a family of general qpsh functions. For each $\lambda$, we assume that $\left\{\varphi_{\lambda, m}\right\}$ is a sequence of ideal functions that converges to $\varphi_{\lambda}$ 
strongly in norm such that $\left\|\varphi_{\lambda}-\varphi_{\lambda, m}\right\|<\frac{1}{m}$. If we set $\psi_{m}=\sup _{\lambda} \varphi_{\lambda, m}$, which is an algebraic qpsh by the previous argument, then $\left\|\psi-\psi_{m}\right\| \leq \frac{1}{m}$ and it follows that $\left\{\psi_{m}\right\}$ is a sequence that converges to $\psi$ strongly in norm.

Since the convex cones QPSH $(X)$ and $\operatorname{QPSH}^{a}(X)$ are closed under taking the supremum by Lemma 4.15, we can introduce the following definition.

Definition 4.16. Let $\varphi$ be a bounded homogeneous function. Assume that the set $\{\psi \in \operatorname{QPSH}(X) \mid \psi \leq \varphi\}$ is nonempty. Then we say the maximal function in this set the qpsh envelope function. We similarly define the algebraic qpsh envelope function of $\varphi$ if it exists.

In general, we cannot ensure the sets defined as above are nonempty. For instance, the function in Example 3.13 is bounded homogeneous but its qpsh envelope function does not exist. Also note that the function $\phi$ in Example 4.7 is qpsh itself but its algebraic qpsh envelope function does not exist.

Lemma 4.17. Let $\varphi$ be a bounded homogeneous function that is determined on some dual complex $\Delta(Y, D)$ in the sense of $\varphi=\varphi \circ r_{Y, D}$. Then, its qpsh envelope function $\psi$ exists. Further, $\psi$ is algebraic qpsh.

Proof. Let $Z \subseteq X$ be the image of the reduced divisor $D$ on $X$, and $\mathfrak{m}$ be the defining ideal of $Z$. Since $\log |\mathfrak{m}|$ is strictly negative on $\Delta(Y, D)$ and $\varphi$ is bounded on $\Delta(Y, D)$, there exists an integer $k$ such that $k \log |\mathfrak{m}| \leq \varphi$ on $\Delta(Y, D)$. Because $\varphi$ is determined on the dual complex $\Delta(Y, D)$ in the sense of $\varphi=\varphi \circ r_{Y, D}$, we have that $k \log |\mathfrak{m}| \leq \varphi$ on $\mathrm{V}_{X}$. It follows that its algebraic qpsh envelope function $\phi$ exists. In particular, its qpsh envelope function $\psi$ exists.

Now we will show that $\psi=\phi$. Set

$$
\mu_{1}=\max _{v \in \Delta(Y, D)}|v(\mathfrak{m})| \quad \text { and } \quad \mu_{2}=\min _{v \in \Delta(Y, D)}|v(\mathfrak{m})| .
$$

For any small number $\varepsilon>0$, we choose $\delta \ll 1$ such that $\left(1+\mu_{1} / \mu_{2}\right) \delta<\varepsilon$ and an ideal function $\phi^{\prime}$ such that $\left\|\phi^{\prime}-\psi\right\|<\delta$. Note that for every valuation $v \in \Delta(Y, D)$ we have

$$
\psi(v)>\phi^{\prime}(v)-\frac{\delta}{\mu_{2}} v(\mathfrak{m}) \geq \phi^{\prime}(v)-\frac{\delta \mu_{1}}{\mu_{2}}>\psi(v)-\left(1+\frac{\mu_{1}}{\mu_{2}}\right) \delta .
$$

We can assume that $\phi^{\prime} \leq \psi$ and $\left|\psi(v)-\phi^{\prime}(v)\right|<\varepsilon$ on $\Delta(Y, D)$ after replacing $\phi^{\prime}$ by $\phi^{\prime}+\left(\delta / \mu_{2}\right) \log |\mathfrak{m}|$. Because $\varphi=\varphi \circ r_{Y, D}$, we obtain that $\phi^{\prime} \leq \varphi$. It follows that $\phi^{\prime} \leq \phi \leq \psi$ by the definition of the qpsh envelope function. Since $\varepsilon$ can be chosen arbitrarily small, this forces $\phi=\psi$ on $\Delta(Y, D)$. If we pick any higher log resolution $\left(Y^{\prime}, D^{\prime}\right)$, we can show that $\phi=\psi$ on $\Delta\left(Y^{\prime}, D^{\prime}\right)$ by the same argument. The conclusion therefore follows from Proposition 3.11.

The above lemma leads to the following definition. 
Definition 4.18. Let $\varphi \in \operatorname{QPSH}(X)$ be a qpsh function. We denote by $\varphi_{Y, D}$ the qpsh envelop function of $\varphi \circ r_{Y, D}$.

Lemma 4.19. Let $\varphi$ be a qpsh function. Then there exists a decreasing sequence of algebraic functions that converges to $\varphi$ strongly in norm.

Proof. Let $\left\{\varphi_{m}\right\}$ be a sequence of ideal functions that converges to $\varphi$ strongly in norm. We can assume that $\varphi_{m}=c_{m} \log \left|\mathfrak{a}_{m}\right|$ and $\left\|\varphi-\varphi_{m}\right\|<\frac{1}{m}$. Let $(Y, D)$ be a $\log$ resolution $\mathfrak{a}_{1}$. It is easy to see that $\left\|\varphi \circ r_{Y, D}-\varphi_{1}\right\|<1$, and therefore $\left\|\varphi_{Y, D}-\varphi_{1}\right\|<1$. We deduce that $\left\|\varphi_{Y, D}-\varphi\right\|<2$. Now we replace $\varphi_{1}$ by $\varphi_{Y, D}$ and continue this process. Note that if $\left(Y^{\prime}, D^{\prime}\right) \succeq(Y, D)$, then $\varphi_{Y^{\prime}, D^{\prime}} \leq \varphi_{Y, D}$ by Proposition 3.11. We easily obtain the required decreasing sequence of algebraic functions.

Lemma 4.20. Let $\left\{\varphi_{m}\right\}$ be a sequence of qpsh functions that converges to a qpsh function $\varphi$ strongly in norm. Then $\mathscr{f}(\varphi)=\mathscr{g}\left((1+\varepsilon) \varphi_{m}\right)$ for a sufficiently small positive real number $\varepsilon>0$ and a sufficiently large integer $m$.

Proof. First we prove that $\mathscr{F}(\varphi) \subseteq \mathscr{F}\left((1+\varepsilon) \varphi_{m}\right)$ for a sufficiently small number $\varepsilon>0$ and a sufficiently large integer $m$. To this end, we pick a sufficiently small number $\varepsilon>0$ such that $\mathscr{f}(\varphi)=\mathscr{F}((1+\varepsilon) \varphi)$. Since $\mathscr{f}((1+\varepsilon) \varphi) \subseteq \mathscr{f}\left((1+\varepsilon) \varphi_{m}\right)$ provided that $m$ is sufficiently large, we have $\mathscr{f}(\varphi) \subseteq \mathscr{g}\left((1+\varepsilon) \varphi_{m}\right)$. Conversely, we pick a sufficiently large integer $m$ such that $\left\|\varphi-\varphi_{m}\right\|<1-\frac{1}{1+\varepsilon}$. Applying Lemma 4.4 again, we see that if $f \in \mathscr{g}\left((1+\varepsilon) \varphi_{m}\right)$ then $\left\|\varphi_{m}\right\|_{f}<\frac{1}{1+\varepsilon}$ and hence $\|\varphi\|_{f} \leq\left\|\varphi_{m}\right\|_{f}+\left\|\varphi-\varphi_{m}\right\|_{f}<1$, which implies that $f \in \mathscr{L}(\varphi)$.

Remark 4.21. Note that we always have $\phi \leq \log |\mathscr{E}(\phi)|$ for an algebraic qpsh function $\phi$ by [JM12, Propositions 6.2 and 6.5]. It follows by Lemmas 4.19 and 4.20 that $\mathscr{F}(\varphi)$ is nonzero and $(1+\varepsilon) \varphi \leq(1+\varepsilon) \varphi_{m} \leq \log |\mathscr{f}(\varphi)|$, where $\left\{\varphi_{m}\right\}$ is a decreasing sequence of algebraic functions that converges to a qpsh function $\varphi$ strongly in norm. Since $\varepsilon$ can be chosen arbitrarily small, we immediately obtain that $\varphi \leq \log |\mathscr{f}(\varphi)|$.

Now we discuss the multiplier ideals of general qpsh functions.

Proposition 4.22. Let $\varphi \in \mathrm{QPSH}(X)$ be a qpsh function on $X$.

(1) Assume that $\psi$ is another qpsh function on X. Then,

$$
\mathscr{F}(\varphi+\psi) \subseteq \mathscr{g}(\varphi) \cdot \mathscr{F}(\psi) .
$$

(2) Assume that $f: X^{\prime} \rightarrow X$ is a regular morphism of schemes. Then,

$$
\mathscr{L}(\varphi) \cdot O_{X^{\prime}}=\mathscr{S}\left(f^{*} \varphi\right) .
$$

Proof. (1) By Lemma 4.19 we can assume that there are decreasing sequences of algebraic functions $\left\{\varphi_{m}\right\}$ and $\left\{\psi_{m}\right\}$ converging to $\varphi$ and $\psi$ strongly in norm 
respectively. Then for some sufficiently large integer $m$, by Lemma 4.20 we have $\mathscr{g}(\varphi+\psi)=\mathscr{g}\left((1+\varepsilon)\left(\varphi_{m}+\psi_{m}\right)\right) \subseteq \mathscr{g}\left((1+\varepsilon) \varphi_{m}\right) \cdot \mathscr{g}\left((1+\varepsilon) \psi_{m}\right)=\mathscr{g}(\varphi) \cdot \mathscr{F}(\psi)$ since $\varphi_{m}+\psi_{m}$ converges to $\varphi+\psi$ strongly in norm. The inclusion appearing in this expression follows from [JM12, Theorem A.2].

(2) Since $f$ is regular, for any ideal function $\phi=\sum_{i} c_{i} \log \mathfrak{a}_{i}$, we have

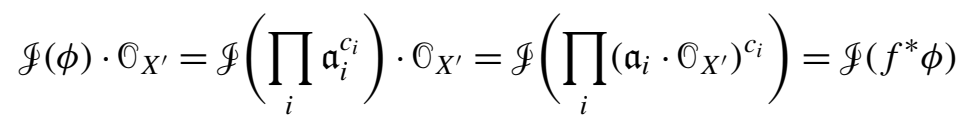

by the argument of [JM12, Proposition 1.9]. If $\left\{\varphi_{m}\right\}$ is a sequence of ideal functions that converges to $\varphi$ strongly in norm, then $f^{*} \varphi_{m}$ is a decreasing sequence of ideal functions that converges to $f^{*} \varphi$ strongly in norm by Proposition 3.2. Therefore we have $\mathscr{f}(\varphi) \cdot O_{X^{\prime}}=\mathscr{F}\left((1+\varepsilon) \varphi_{m}\right) \cdot O_{X^{\prime}}=\mathscr{F}\left((1+\varepsilon) f^{*} \varphi_{m}\right)=\mathscr{F}\left(f^{*} \varphi\right)$.

Recall from [JM12] that if $\mathfrak{b}_{\bullet}$ is subadditive, then the limit

$$
v\left(\mathfrak{b}_{\bullet}\right):=\lim _{m \rightarrow \infty} \frac{1}{m} v\left(\mathfrak{b}_{m}\right) \in[0,+\infty]
$$

is well-defined. For the purpose of constructing a "good" valuative function, we introduce the notion of a subadditive sequence of ideals of controlled growth as follows.

Definition 4.23 [JM12, Definition 2.9]. A subadditive sequence of ideals $\mathfrak{b}_{\bullet}$ is of controlled growth if

$$
\frac{v\left(\mathfrak{b}_{t}\right)}{t}>v\left(\mathfrak{b}_{\bullet}\right)-\frac{A(v)}{t}
$$

for every nontrivial tempered valuation $v$ and every $t>0$.

We see that $v\left(\mathfrak{b}_{\bullet}\right):=\lim _{m \rightarrow \infty} \frac{1}{m} v\left(\mathfrak{b}_{m}\right)<+\infty$ for every nontrivial tempered valuation $v$. Furthermore, if we define $\log \left|\mathfrak{b}_{\bullet}\right|(v)=-v\left(\mathfrak{b}_{\bullet}\right)$, then $\log \left|\mathfrak{b}_{\bullet}\right|$ is approximated by $\frac{1}{m} \log \left|\mathfrak{b}_{m}\right|$ strongly in norm and hence qpsh. Given a qpsh function, if we define $\mathscr{G}(\varphi)_{t}:=\mathscr{F}(t \varphi)$, then $\mathscr{F}(\varphi)$. is a subadditive sequence of controlled growth by Proposition 4.22, Definition 4.1 and Remark 4.2. This allows us to give a characterization of qpsh functions as follows. Readers could compare the following theorem with Theorem 4.12.

Theorem 4.24. If $\varphi$ is a bounded homogeneous function, the following statements are equivalent.

(1) $\varphi$ is qpsh.

(2) There is a subadditive sequence of ideals $\mathfrak{b}_{\bullet}$ of controlled growth such that $\varphi=\log \left|\mathfrak{b}_{\bullet}\right|$.

(3) The ideal $\mathscr{f}(t \varphi)$ is nonzero for every $t>0$ and $\varphi=\log |\Phi(\varphi)$.$| .$ 
Proof. If we assume (1), then (3) follows from the previous argument together with Definition 4.1 and Remark 4.2. Note that (3) implies (2) if we simply put $\mathfrak{b}_{\bullet}=\mathscr{g}(\varphi)$. Finally, (1) follows from (2) by the previous argument.

Remark 4.25. From the theorem we see that every qpsh function $\varphi$ can be approximated by a decreasing sequence of ideal functions $\varphi_{k}$ in norm. Indeed, we can take $\varphi_{k}=\frac{1}{2^{k}} \log \left|\mathscr{f}\left(2^{k} \varphi\right)\right|$. However, if $\varphi$ is only the pointwise limit of a decreasing sequence of ideal functions on $\mathrm{V}_{X}$, then $\varphi$ is not necessarily qpsh (see Example 3.13).

An immediate application of the preceding discussion is the following result on the support of a qpsh function.

Corollary 4.26. Let $\varphi$ be a qpsh function. Then its support $\operatorname{supp} \varphi$ is a countable union of proper Zariski closed subsets of $X$.

Remark 4.27. Readers can compare the constructions here with [Boucksom et al. 2008]. If we work on $X=\operatorname{Spec} \widehat{R}$, where $R$ is the localization of $\mathbb{C}\left[x_{1}, \ldots, x_{n}\right]$ at the origin, then our definition of qpsh functions coincides the notion of formal psh functions. A brief argument is as follows. Given a formal psh function $g$, we have a subadditive sequence of ideals $\left\{\mathscr{L}^{2}(\operatorname{tg})\right\}_{t>0}$ in $\widehat{R}$, that satisfies $v\left(\mathscr{L}^{2}(\operatorname{tg})\right)+$ $A(v)+(1+\epsilon) g(v) \geq 0$ for every quasimonomial valuation $v$ centred at the origin and an arbitrarily small $\epsilon$; see [Boucksom et al. 2008, Theorems 3.10 and 3.9]. It follows that $\left\{\mathscr{L}^{2}(t g)\right\}_{t>0}$ form a subadditive sequence of ideals of controlled growth that induces to a qpsh function $\varphi$ on $X$. Therefore $\varphi(v)=g(v)$ for every divisorial valuation $v$ centred at the origin. Conversely, a qpsh function can be naturally viewed as a formal psh function by definition. Therefore we construct an one-to-one correspondence. The details are left to the readers.

Remark 4.28. Recall from complex geometry that a function $\varphi: X \rightarrow[-\infty,+\infty)$ from a complex manifold is qpsh if it is locally equal to the sum of a smooth function and a psh function. If $X$ is a smooth complex variety, we should be able to define the valuative transform of $\varphi$, which is expected to be a qpsh function on the valuation space $\mathrm{V}_{X}$ as defined in this paper. This was done locally in [Boucksom et al. 2008] and its predecessors [Favre and Jonsson 2004; 2005a; 2005b]. However, the global situation is not fully understood by us at this point.

\section{Computing norms}

\section{Generalities.}

Definition 5.1. Let $\varphi$ be a bounded homogeneous function and $\mathfrak{q}$ be a nonzero ideal on $X$. We say a nontrivial tempered valuation $v \in \mathrm{V}_{X}^{*}$ computes $\|\varphi\|_{\mathfrak{q}}$ if the equality $\|\varphi\|_{\mathfrak{q}}=|\varphi(v)| /(A(v)+v(\mathfrak{q}))$ holds. 
The main result of this section is the following theorem.

Theorem 5.2. Let $\varphi \in \operatorname{QPSH}(X)$ be a qpsh function and let $\mathfrak{q}$ be a nonzero ideal on $X$. Then there exists a nontrivial tempered valuation $v$ that computes $\|\varphi\|_{\mathfrak{q}}$.

Before we prove this theorem, we need some preparations.

Proposition 5.3. Let $\varphi$ be a bounded homogeneous function that is determined on some dual complex $\Delta(Y, D)$ in the sense of $\varphi=\varphi \circ r_{Y, D}$. Assume that $\varphi$ is weakly continuous (see Definition 3.4). Then there exists a quasimonomial valuation $v$ that computes $\|\varphi\|_{\mathfrak{q}}$. If we assume further that $\varphi$ is affine on each face of $\Delta(Y, D)$, then there exists a divisorial valuation $v$ that computes $\|\varphi\|_{\mathfrak{q}}$.

Proof. For every nontrivial tempered valuation $v \in V_{X}^{*}$, we have

$$
\frac{|\varphi(v)|}{A(v)+v(\mathfrak{q})} \geq \frac{\left|\varphi \circ r_{Y, D}(v)\right|}{A\left(r_{Y, D}(v)\right)+r_{Y, D}(v)(\mathfrak{q})}
$$

with equality if and only if $v \in \mathrm{QM}(Y, D)$. Thus

$$
\|\varphi\|_{\mathfrak{q}}=\sup _{v \in \mathrm{QM}(Y, D)} \frac{|\varphi(v)|}{A(v)+v(\mathfrak{q})}=\sup _{v \in \Delta(Y, D)} \frac{|\varphi(v)|}{1+v(\mathfrak{q})} .
$$

Since $\varphi$ is weakly continuous, the function $v \rightarrow|\varphi(v)| /(A(v)+v(\mathfrak{q}))$ is continuous on $\operatorname{QM}(Y, D)$. Therefore the function $v \rightarrow|\varphi(v)| /(1+v(\mathfrak{q}))$ is continuous on the dual complex $\Delta(Y, D)$ and hence achieves its maximum in $\Delta(Y, D)$.

Assume that $\varphi$ is affine on $\Delta(Y, D)$, and denote by $\left\{D_{i}\right\}$ the irreducible components of $D$. After replacing $(Y, D)$ by some higher log resolution, we can assume that $(Y, D)$ is a $\log$ resolution of $\mathfrak{q}$ by Lemma 3.8. Then we have $\|\varphi\|_{\mathfrak{q}}=$ $\max _{D_{i}}\left(\left|\varphi\left(\operatorname{ord}_{D_{i}}\right)\right| /\left(A\left(\operatorname{ord}_{D_{i}}\right)+\operatorname{ord}_{D_{i}}(\mathfrak{q})\right)\right)$, where $D_{i}$ runs over all irreducible components of $D$ since the functions $\varphi, A$ and $\log |\mathfrak{q}|$ are all affine on $\Delta(Y, D)$.

Computing norms of qpsh functions. This subsection is devoted to the proof of Theorem 5.2. The proof here follows the strategy of [JM12]. We first consider the local case.

Lemma 5.4. Let $(R, \mathfrak{m})$ be a local ring, let $\varphi \in \mathrm{QPSH}(\operatorname{Spec} R)$ be a qpsh function, and let $\mathfrak{q}$ be a nonzero ideal on $\operatorname{Spec} R$. We set $\lambda^{-1}=\|\varphi\|_{\mathfrak{q}}$ and assume that $\sqrt{(\mathscr{F}(\lambda \varphi): \mathfrak{q})}=\mathfrak{m}$. If we define another qpsh function $\psi=\max \{\varphi, p \log |\mathfrak{m}|\}$ for a sufficiently large integer $p$, then $\|\varphi\|_{\mathfrak{q}}=\|\psi\|_{\mathfrak{q}}$. Moreover, if a nontrivial tempered valuation $v$ computes $\|\psi\|_{\mathfrak{q}}$, then $v$ also computes $\|\varphi\|_{\mathfrak{q}}$.

Proof. Since $\sqrt{(\mathscr{F}(\lambda \varphi): \mathfrak{q})}=\mathfrak{m}$, we have $\mathfrak{m}^{n} \cdot \mathfrak{q} \subseteq \mathscr{g}(\lambda \varphi)$ for some integer $n$. Set $\lambda^{\prime-1}=\|\varphi\|_{\mathfrak{m}^{n} \cdot \mathfrak{q}}$, it follows that $\lambda^{\prime}>\lambda$ by Lemma 4.4. Pick an integer $p>n /\left(\lambda^{\prime}-\lambda\right)$, and fix a sufficiently small number $\varepsilon \ll 1$ such that $p>n /\left((1-\varepsilon) \lambda^{\prime}-\lambda\right)$. Observe that

$$
\|\psi\|_{\mathfrak{q}}=\sup _{v \in \mathrm{V}_{R}^{*}} \frac{\min \{-\varphi(v), p v(\mathfrak{m})\}}{A(v)+v(\mathfrak{q})} \geq \sup _{v \in \mathrm{V}_{\varepsilon}^{*}} \frac{\min \{-\varphi(v), p v(\mathfrak{m})\}}{A(v)+v(\mathfrak{q})},
$$


where $\mathrm{V}_{\varepsilon}^{*}$ is the set of $v \in \mathrm{V}_{R}^{*}$ satisfying $-\varphi(v) /(A(v)+v(\mathfrak{q})) \geq(1-\varepsilon) / \lambda$.

By the definition of $\lambda^{\prime}$ we have $n v(\mathfrak{m}) /(-\varphi(v)) \geq \lambda^{\prime}-(A(v)+v(\mathfrak{q})) /(-\varphi(v))$ for every nontrivial tempered valuation $v$. This implies that

$$
\begin{aligned}
\|\psi\|_{\mathfrak{q}} & \geq \sup _{v \in \mathrm{V}_{\varepsilon}} \frac{-\varphi(v)}{A(v)+v(\mathfrak{q})} \min \left\{1, \frac{p}{n}\left(\lambda^{\prime}-\frac{A(v)+v(\mathfrak{q})}{-\varphi(v)}\right)\right\} \\
& \geq \sup _{v \in \mathrm{V}_{\varepsilon}} \frac{-\varphi(v)}{A(v)+v(\mathfrak{q})} \min \left\{1, \frac{p}{n}\left(\lambda^{\prime}-\frac{\lambda}{1-\varepsilon}\right)\right\}=\sup _{v \in \mathrm{V}_{\varepsilon}} \frac{-\varphi(v)}{A(v)+v(\mathfrak{q})}=\|\varphi\|_{\mathfrak{q}} .
\end{aligned}
$$

Moreover, if a nontrivial tempered valuation $v$ computes $\|\psi\|_{\mathfrak{q}}$, we see from these inequalities that $v$ also computes $\|\varphi\|_{\mathfrak{q}}$.

Lemma 5.5. Let $(R, \mathfrak{m})$ be a local ring, let $\varphi$ be an ideal function on $\mathrm{Spec} R$ such that $\varphi \geq p \log |\mathfrak{m}|$ for some integer $p$, and let $\mathfrak{q}$ be a nonzero ideal on $\mathrm{Spec} R$. Then there exists a nontrivial tempered valuation $v \in \mathbb{V}_{R, \mathfrak{m}, M}$ (see Definition 2.6) that computes $\|\psi\|_{\mathfrak{q}}$ provided that $M>p \cdot\|\varphi\|_{\mathfrak{q}}^{-1}$.

Proof. If we write $c=p / M$, then $0<c<\|\psi\|_{\mathfrak{q}}$. For every $v \in \mathbb{V}_{R, \mathfrak{m}}$ such that $-\varphi(v) /(A(v)+v(\mathfrak{q}))>c$, we have $A(v) \leq A(v)+v(\mathfrak{q})<p / c=M$. Thus $\|\varphi\|_{\mathfrak{q}}=\sup v \in \mathbb{V}_{R, \mathfrak{m}, M}-\varphi(v) /(A(v)+v(\mathfrak{q}))$. Note that $\mathbb{V}_{R, \mathfrak{m}, M}$ is compact. Since the function $v \rightarrow-\varphi(v) /(A(v)+v(\mathfrak{q}))$ is usc as the valuative function $A$ is lsc, the maximum can be achieved in $\mathbb{V}_{R, \mathfrak{m}, M}$.

Lemma 5.6. Let $\varphi \in \mathrm{QPSH}(X)$ be a qpsh function on $X$ and $\left\{\varphi_{m}\right\}$ be a decreasing sequence of algebraic functions converging to $\varphi$ strongly in norm. Set $\lambda^{-1}=\|\varphi\|_{\mathfrak{q}}$ and $\lambda_{m}^{-1}=\left\|\varphi_{m}\right\|_{\mathfrak{q}}$. Then, $\mathscr{f}(\lambda \varphi) \subseteq \mathscr{F}\left(\lambda_{m} \varphi_{m}\right)$ for every sufficiently large integer $m$.

Proof. If $f \in \mathscr{J}(\lambda \varphi)$, then $\|\varphi\|_{f}<(1-\varepsilon) / \lambda$ for a sufficiently small number $\varepsilon>0$. We have $\left\|\varphi_{m}\right\|_{f} \leq\|\varphi\|_{f}<(1-\varepsilon) / \lambda<\lambda_{m}^{-1}$ since $\lambda_{m}<\lambda /(1-\varepsilon)$ for sufficiently large $m$. It follows that $f \in \mathscr{f}\left(\lambda_{m} \varphi_{m}\right)$ by Lemma 4.4.

Lemma 5.7. Let $(R, \mathfrak{m})$ be a local ring, let $\varphi$ be a qpsh function on $\operatorname{Spec} R$ such that $\varphi \geq p \log |\mathfrak{m}|$, and let $\mathfrak{q}$ be a nonzero ideal on $\operatorname{Spec} R$. Then there exists a nontrivial tempered valuation $v \in \mathbb{V}_{R, \mathfrak{m}, M}$ which computes $\|\varphi\|_{\mathfrak{q}}$ provided that $M>p \cdot\|\varphi\|_{\mathfrak{q}}^{-1}$.

Proof. Assume that $\left\{\varphi_{m}\right\}$ is a decreasing sequence of ideal functions which converges to $\psi$ strongly in norm. Then $\mathfrak{m}^{n} \cdot \mathfrak{q} \subseteq \mathscr{g}(\lambda \varphi) \subseteq \mathscr{F}\left(\lambda_{m} \varphi_{m}\right)$ for every sufficiently large

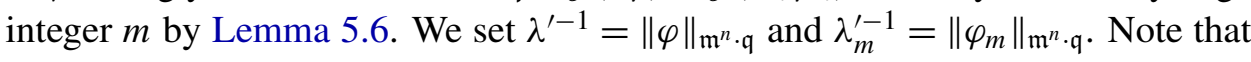
$M>p \cdot \lambda_{m}$ for every sufficiently large integer $m$. Therefore for every sufficiently large integer $m$, there exists $v_{m} \in \mathbb{V}_{R, \mathfrak{m}, M}$ that computes $\left\|\varphi_{m}\right\|_{\mathfrak{q}}$ by Lemma 5.5. By passing $\left\{\varphi_{m}\right\}$ to a subsequence, we can assume $\left\{v_{m}\right\}$ is a sequence of points that 
converges to some point $v \in \mathbb{V}_{R, \mathfrak{m}, M}$. Note that

$$
\begin{aligned}
\frac{-\lambda \varphi(v)}{A(v)+v(\mathfrak{q})} \geq \frac{-\lambda \varphi_{m}(v)}{A(v)+v(\mathfrak{q})} & \geq \frac{-\lambda \varphi_{m}\left(v_{n}\right)}{A\left(v_{n}\right)+v_{n}(\mathfrak{q})}-\delta \\
& \geq 1-\left\|\lambda \varphi_{m}-\lambda_{n} \varphi_{n}\right\|_{\mathfrak{q}}-\delta \\
& \geq 1-\lambda\left\|\varphi_{m}-\varphi_{n}\right\|_{\mathfrak{q}}-\delta-\left(\lambda_{n}-\lambda\right)\|\varphi\|_{\mathfrak{q}},
\end{aligned}
$$

where the second inequality holds because the function $v \rightarrow-\lambda \varphi_{m}(v) /(A(v)+v(\mathfrak{q}))$ is usc. Since $\left\|\psi_{m}-\psi_{n}\right\|_{\mathfrak{q}}, \delta$ and $\lambda_{n}-\lambda$ can be chosen arbitrarily small, we have $-\lambda \psi(v) /(A(v)+v(\mathfrak{q})) \geq 1$ and the conclusion follows.

Now we turn to treat the global case.

Proof of Theorem 5.2. Pick a generic point $\xi$ of $V(\mathscr{E}(\lambda \varphi): \mathfrak{q})$. Note that by Lemma 4.5(3) $\|\varphi\|_{\mathfrak{q}}=\left\|\varphi_{\xi}\right\|_{\mathfrak{q} \cdot \mathscr{O}_{X, \xi}}$. After replacing $X$ and $\varphi$ by Spec $\mathcal{O}_{X, \xi}$ and $\varphi_{\xi}$, respectively, we reduce the global case to the local case. After replacing $\varphi$ by $\max \{\varphi, p \log |\mathfrak{m}|\}$ for a sufficiently large integer $p$ by Lemma 5.4, we can assume that $\varphi \geq p \log |\mathfrak{m}|$. Finally by Lemma 5.7, there exists a valuation $v \in \mathbb{V}_{X, \xi, M}$ that computes $\|\varphi\|_{\mathfrak{q}}$.

An immediate consequence of Theorem 5.2 is the following corollary.

Corollary 5.8. Let $\varphi$ be a qpsh function on $X$. Then, on every open subset $U$, we can explicitly write

$$
\Gamma(U, \mathscr{g}(\varphi))=\left\{f \in \Gamma\left(U, O_{X}\right) \mid v(f)+A(v)+\varphi(v)>0 \text { for every } v \in \mathrm{V}_{U}^{*}\right\} .
$$

Let $\mathfrak{q}$ be a nonzero ideal on $X$. Then, $\mathfrak{q} \subseteq \mathscr{g}(\varphi)$ if and only if $v(\mathfrak{q})+A(v)+\varphi(v)>0$ for every $v \in \mathrm{V}_{X}^{*}$.

The following conjecture was raised as [JM12, Conjecture B] (cf. [JM12, Theorem 7.8]). It is already known for several special cases (see [JM12, Sections 8 and 9]).

Conjecture 5.9. Let $\varphi$ be a qpsh function on $X$ and $\mathfrak{q}$ be a nonzero ideal on $X$. Then there exists a nontrivial quasimonomial valuation $v$ which computes $\|\varphi\|_{\mathfrak{q}}$. Conversely, if a nontrivial tempered valuation $v$ computes the norm of some qpsh function, then $v$ is quasimonomial.

\section{Applications}

If $X$ is a smooth complex projective variety, we are interested in associating a qpsh function to a line bundle that plays the role of a semipositive singular metric. The starting point is the following easy observation. Given a pseudo-effective line bundle $L$, an ideal $\mathfrak{a}$ together with a nonnegative rational number $\lambda$ such that $L \otimes \mathfrak{a}^{\lambda}$ is semi-ample corresponds to a semipositive singular metric $h$ in the sense that 
they give the same multiplier ideals $\mathscr{g}\left(\mathfrak{a}^{\lambda m}\right)=\mathscr{g}\left(h^{\otimes m}\right)$ for every integer $m>0$. However in general, this correspondence becomes quite mysterious since many analogue notions cannot be constructed. This has been studied in many relevant references [Boucksom 2004; Ein et al. 2006; 2009; Ein and Popa 2008; Lehmann 2011; Nakayama 2004]. We will discuss the qpsh function associated to a line bundle in detail within this section. Besides, it might be possible to generalize the results in this section to varieties with mild singularities such as klt singularities (see [Boucksom et al. 2012d; Boucksom et al. 2013b]).

Throughout this section $X$ will be a projective smooth variety over $\mathbb{C}$ for simplicity. The term "divisor" will always refer to a $\mathbb{Q}$-Cartier $\mathbb{Q}$-divisor. Given a section $s \in H^{0}(X, L)$ of a line bundle, the notation $\log |s|$ denotes the qpsh function defined locally by a regular function corresponding to $s$.

\section{D-psh functions.}

Definition 6.1. Let $D$ be a divisor. We define the set $\mathscr{L}_{D}:=\left\{\frac{\log |\mathfrak{a}|}{k} \mid k m D \otimes \mathfrak{a}^{m}\right.$ is globally generated for every sufficiently divisible $\left.m\right\}$.

We then define set of $D$-psh functions to be the closure $\operatorname{PSH}(D)=\overline{\mathscr{L}_{D}}$ in norm.

Lemma 6.2. (1) $\mathrm{PSH}(D)$ is compact and convex in $\mathrm{QPSH}(X)$.

(2) $\operatorname{PSH}(t D)=t \operatorname{PSH}(D)$ for any $t \in \mathbb{Q}_{>0}$.

(3) $\operatorname{PSH}(D)+\operatorname{PSH}\left(D^{\prime}\right) \subseteq \operatorname{PSH}\left(D+D^{\prime}\right)$.

(4) If $A$ is a semiample divisor, then $\operatorname{PSH}(D) \subseteq \operatorname{PSH}(D+A)$.

Proof. We firstly prove (1). To prove that $\operatorname{PSH}(D)$ is convex, it suffices to show that $\mathscr{L}_{D}$ is convex. Given qpsh functions $\varphi, \varphi^{\prime} \in \mathscr{L}_{D}$ and a rational number $0<\lambda<1$, we will show that $\lambda \varphi+(1-\lambda) \varphi^{\prime} \in \mathscr{L}_{D}$. If we write $\varphi=\frac{1}{k} \log |\mathfrak{a}|, \varphi^{\prime}=\frac{1}{k^{\prime}} \log \left|\mathfrak{a}^{\prime}\right|$ and $\lambda=q / p$, then

$$
\begin{aligned}
\lambda \varphi+(1-\lambda) \varphi^{\prime} & =\frac{1}{k p} \log \left|\mathfrak{a}^{q}\right|+\frac{1}{k^{\prime} p} \log \left|\mathfrak{a}^{\prime p-q}\right| \\
& =\frac{1}{k k^{\prime} p} \log \left|\mathfrak{a}^{q k^{\prime}} \cdot \mathfrak{a}^{\prime(p-q)}\right| .
\end{aligned}
$$

It is easy to check that $k k^{\prime} p m L \otimes \mathfrak{a}^{m q k^{\prime}} \cdot \mathfrak{a}^{\prime m k(p-q)}$ is globally generated for every sufficiently divisible integer $m$ and the conclusion follows. Note that (2), (3) and (4) can be proved in a similar way.

Question 6.3. Let $\varphi$ be a general qpsh function. Does there exist a divisor $D$ such that $\varphi \in \operatorname{PSH}(D)$ ? 
Definition 6.4. For an ample divisor $A$, the set of pseudo $D$-psh functions is defined to be $\operatorname{PSH}_{\sigma}(D):=\bigcap_{\varepsilon>0} \operatorname{PSH}(D+\varepsilon A)$.

Note that this definition is independent of the choice of the ample divisor $A$, and that the set $\mathrm{PSH}_{\sigma}(D)$ also satisfies the properties listed in Lemma 6.2.

Theorem 6.5 (Nadel vanishing). Let $L$ be a line bundle on a smooth projective variety $X$ and $L \equiv A+D$, where $A$ is a nef and big $\mathbb{Q}$-divisor. Assume that $\varphi \in \operatorname{PSH}_{\sigma}(D)$. Then

$$
H^{i}\left(X,\left(K_{X}+L\right) \otimes \mathscr{g}(\varphi)\right)=0
$$

for all $i>0$.

Proof. By Kodaira's lemma, $A-\delta E$ is ample for some effective divisor $E$ and every sufficiently small number $\delta>0$. If we write $\varphi_{E}=\log \left|O_{X}(-E)\right|$, then by semicontinuity of multiplier ideals we have $\mathscr{f}(\varphi)=\mathscr{\Phi}\left(\varphi+\delta \varphi_{E}\right)$ for every sufficiently small number $\delta>0$. After replacing $A$ and $\varphi$ with $A-\delta E$ and $\varphi+\delta \varphi_{E}$, respectively, we can assume that $A$ is ample.

By definition we can assume that there exists a sequence of ideal functions $\left\{\varphi_{k}\right\}$ that converges to $\varphi$ strongly in norm, such that $\varphi_{k} \in \mathscr{L}_{D+\epsilon_{k} A}$ and $\epsilon_{k} \rightarrow 0^{+}$. Choose $\varepsilon \ll 1$ such that $A-\varepsilon D$ is ample. We see that $\mathscr{f}(\varphi)=\mathscr{f}\left((1+\varepsilon) \varphi_{k}\right)$ for every sufficiently large integer $k$ by Lemma 4.20 . Note that $(1+\varepsilon) \varphi_{k} \in \mathscr{L}_{(1+\varepsilon)\left(D+\epsilon_{k} A\right)}$. For a sufficiently large integer $k, A-\varepsilon D-(1+\varepsilon) \epsilon_{k} A$ is ample. After replacing $A$ and $\varphi$ by $A-\varepsilon D-(1+\varepsilon) \epsilon_{k} A$ and $(1+\varepsilon) \varphi_{k}$, respectively, we reduce to the classical Nadel vanishing (see [Lazarsfeld 2004]).

As an application of this theorem, one can easily deduce the following theorem by letting $G=K_{X}+(n+1) H$, where $H$ is a hypersurface of $X$ and $n=\operatorname{dim} X$, with the aid of the Castelnuovo-Mumford regularity.

Theorem 6.6 (global generation). Let $D$ be a divisor on $X$. A qpsh function $\varphi$ lies in $\operatorname{PSH}_{\sigma}(D)$ if and only if there exists a line bundle $G$ such that $(m D+G) \otimes \mathscr{f}(m \varphi)$ is globally generated for all $m \in \mathbb{Z}_{+}$with $m D$ integral.

Given a qpsh function $\varphi$, a positive real number $\lambda$ is said to be the (higher) jumping number of $\varphi$ if $\mathscr{f}((\lambda-\epsilon) \varphi) \supsetneq \mathscr{\&}(\lambda \varphi)$ for every positive real number $\epsilon$.

Definition 6.7. Let $\varphi$ be a qpsh function. We define the ideal $\mathscr{f}_{-}(\varphi)$ to be the largest ideal in the set $\left\{\mathfrak{a}|\|\log |\mathfrak{a}|-\varphi\| \leq 1\}\right.$. One can see that $\mathscr{F}_{-}(\varphi)$ can be written explicitly as

$$
\Gamma\left(U, \mathscr{g}_{-}(\varphi)\right)=\left\{f \in \mathcal{O}_{X}(U) \mid v(f)+A(v)+\varphi(v) \geq 0 \text { for every } v \in V_{U}^{*}\right\}
$$

for every open subset $U$. 
Lemma 6.8. If $\varphi$ is D-psh for some divisor $D$, then the descending chain of ideals $\mathscr{g}((1-\epsilon) \varphi)$ stabilizes as $\epsilon \rightarrow 0^{+}$. Further, $\mathscr{f}((1-\epsilon) \varphi)=\mathscr{g}_{-}(\varphi)$ for $\epsilon \ll 1$. It follows that the set of its (higher) jumping numbers is discrete.

Proof. By adding an ample divisor to $D$, we can assume that $D$ is Cartier. By Theorem 6.5 and the Castelnuovo-Mumford regularity there exists an ample line bundle $G$ such that $O_{X}(D+G) \otimes \mathscr{F}((1-\epsilon) \varphi)$ is globally generated for $\epsilon \ll 1$. Since the descending chain of vector spaces $H^{0}\left(X, \mathcal{O}_{X}(D+G) \otimes \mathscr{g}((1-\epsilon) \varphi)\right)$ will stabilize as $\epsilon \rightarrow 0^{+}$, the descending chain of ideals $\mathscr{\mathscr { S }}((1-\epsilon) \varphi)$ will stabilize. The reader can find more details in [Lehmann 2011, Theorem 4.2].

Fix a sufficiently small number $\epsilon^{\prime}$. Since $\left\|\log \left|\mathscr{g}\left(\left(1-\epsilon^{\prime}\right) \varphi\right)\right|-(1-\epsilon) \varphi\right\|<1$ for every sufficiently small number $\epsilon$, we see that $\left\|\log \left|\mathscr{g}\left(\left(1-\epsilon^{\prime}\right) \varphi\right)\right|-\varphi\right\| \leq 1$. It follows that $\mathscr{f}((1-\epsilon) \varphi) \subseteq \mathscr{F}_{-}(\varphi)$. To prove the converse inclusion, simply notice that

$$
\Gamma\left(U, \mathscr{F}_{-}(\varphi)\right)=\left\{f \in \mathcal{O}_{X}(U) \mid v(f)+A(v)+\varphi(v) \geq 0 \text { for every } v \in V_{U}^{*}\right\}
$$

and hence $\mathscr{f}((1-\epsilon) \varphi) \supseteq \mathscr{F}_{-}(\varphi)$ for $\epsilon \ll 1$ by Corollary 5.8.

To investigate the structure of the sets $\operatorname{PSH}(D)$ and $\operatorname{PSH}_{\sigma}(D)$, we need the following construction. Given an integer $k$, a divisor $D$ and a qpsh function $\varphi$, we define the linear system $V_{m}(D, \varphi, t):=\left\{L \in|\llcorner m D\lrcorner|\left|\frac{1}{m} \log \right| s_{L}\left|\leq \frac{1}{t} \log \right| \mathscr{F}_{-}(t \varphi) \mid\right\}$, where $s_{L}$ is the section associated to the divisor $L$ and $\epsilon \ll 1$. If we choose $\mathfrak{a}(D, \varphi, t)_{m}:=\mathfrak{b}\left(V_{m}(D, \varphi, t)\right)$, the base ideal of the linear system $V_{m}(D, \varphi, t)$, then $\mathfrak{a}(D, \varphi, t)$. is a graded sequence of ideals. Moreover, for every positive rational number $t$, we define $\varphi_{t}^{D}:=\log |\mathfrak{a}(D, \varphi, t) \bullet|$.

Lemma 6.9. Let $D$ be a divisor on $X$ and $\varphi$ be a qpsh function. Then, $\varphi \in \operatorname{PSH}(D)$ if and only if $\varphi=\lim _{t \rightarrow \infty} \varphi_{t}^{D}$ pointwise.

Proof. First assume that $\varphi \in \operatorname{PSH}(D)$. Let $\left\{\varphi_{m}\right\}$ be a sequence of ideal functions that converges to $\varphi$ such that each $\varphi_{m} \in \mathscr{L}_{D}$. If $t$ is not a (higher) jumping number of $\varphi$, then, by Lemma 4.20 we have

$$
\mathscr{F}_{-}(t \varphi)=\mathscr{g}((t-\epsilon) \varphi)=\mathscr{F}\left(\left(t-\epsilon+\epsilon^{\prime}\right) \varphi_{m}\right) \supseteq \mathscr{F}_{-}\left(t \varphi_{m}\right)
$$

and

$$
\mathscr{J}_{-}(t \varphi)=\mathscr{F}(t \varphi)=\mathscr{F}\left((t+\epsilon) \varphi_{m}\right) \subseteq \mathscr{F}_{-}\left(t \varphi_{m}\right)
$$

for every sufficiently large integer $m$. It follows that $\mathscr{g}_{-}(t \varphi)=\mathscr{f}_{-}\left(t \varphi_{m}\right)$ and $\varphi_{t}^{D}=\varphi_{m, t}^{D}$. Note that $\varphi_{m . t}^{D} \geq \varphi_{m}$, and hence $\frac{1}{t} \log \left|\mathscr{F}_{-}(t \varphi)\right| \geq \varphi_{t}^{D} \geq \varphi$. If $t$ is a (higher) jumping number, then $\varphi_{t}^{D} \geq \varphi_{t-\epsilon}^{D} \geq \varphi$. Therefore, we have $\left\|\varphi_{t}^{D}-\varphi\right\| \leq \frac{1}{t}$ and hence $\varphi=\lim _{t \rightarrow \infty} \varphi_{t}^{D}$.

Conversely, we assume that $\varphi=\lim _{t \rightarrow \infty} \varphi_{t}^{D}$. Since $\varphi_{t}^{D}$ is algebraic from $\mathfrak{a}(D, \varphi, t)$ • for each $t, \varphi_{t}^{D}$ is $D$-psh for every $t>0$. Since $\frac{1}{t} \log \left|\mathscr{F}_{-}(t \varphi)\right| \geq \varphi_{t}^{D}$ and $\varphi_{t}^{D}$ has a 
decreasing subsequence, $\varphi_{t}^{D}$ converges to $\varphi$ strongly in norm, which implies the conclusion immediately.

For every nontrivial tempered valuation $v$, we define $v(\|D\|)=v\left(\mathfrak{a}_{\bullet}\right)$, where $\mathfrak{a}_{m}=\mathfrak{b}(|\llcorner m D\lrcorner|)$.

Proposition 6.10. The set $\mathrm{PSH}(D)$ is closed under taking the supremum. The maximal D-psh function $\varphi_{\max }$ can be written explicitly as $\varphi_{\max }(v)=-v(\|D\|)$ for all $v \in \mathrm{V}_{X}^{*}$.

Proof. Let $\varphi_{\lambda}$ be a family of $D$-psh functions. By Lemma $6.9 \varphi_{\lambda}=\lim _{t \rightarrow \infty} \varphi_{\lambda, t}^{D}$. Note that $\varphi_{\lambda, t}^{D}=\log \left|\mathfrak{a}\left(D, \varphi_{\lambda}, t\right) \bullet\right|$, where $\mathfrak{a}\left(D, \varphi_{\lambda}, t\right)_{m}=\mathfrak{b}\left(V_{m}\left(D, \varphi_{\lambda}, t\right)\right)$.

If we write $\varphi=\sup _{\lambda} \varphi_{\lambda}$, then $\mathscr{g}_{-}\left(t \varphi_{\lambda}\right) \subseteq \mathscr{g}_{-}(t \varphi)$ for every $\lambda$ and every $t$. It follows that $\mathfrak{b}\left(V_{m}\left(D, \varphi_{\lambda}, t\right)\right) \subseteq \mathfrak{b}\left(V_{m}(D, \varphi, t)\right)$ for every $m, \lambda$ and $t$. We deduce that $\sup _{\lambda} \varphi_{\lambda, t}^{D} \leq \varphi_{t}^{D}$ and hence

$$
\varphi(v)=\sup _{\lambda} \lim _{t \rightarrow \infty} \varphi_{\lambda, t}^{D}(v) \leq \lim _{t \rightarrow \infty} \sup _{\lambda} \varphi_{\lambda, t}^{D}(v) \leq \lim _{t \rightarrow \infty} \varphi_{t}^{D}(v)
$$

for every $v \in \mathrm{V}_{X}^{*}$. Note that the pointwise limits appearing in these inequalities exist because we can take decreasing subsequences which are bounded from below. Since $\frac{1}{t} \log \left|\mathscr{F}_{-}(t \varphi)\right| \geq \varphi_{t}^{D}$, we obtain the equality $\varphi=\lim _{t \rightarrow \infty} \varphi_{t}^{D}$ and $\varphi$ is $D$-psh by Lemma 6.9.

Now we prove that $\varphi_{\max }(v)=-v(\|D\|)$ for all $v \in V_{X}^{*}$. Let $\varphi$ be a qpsh function such that $\varphi(v)=-v(\|D\|)$. Because $\varphi$ is algebraic from $\mathfrak{a}_{\bullet}$, where $\mathfrak{a}_{m}=\mathfrak{b}(|\llcorner m D\lrcorner|), \varphi$ is $D$-psh. It suffices to show that $\varphi_{\max } \leq \varphi$. For each $t, \varphi_{\max , t}^{D}=\log \left|\mathfrak{a}\left(D, \varphi_{\max }, t\right) \bullet\right|$, where $\mathfrak{a}\left(D, \varphi_{\max }, t\right)_{m}=\mathfrak{b}\left(V_{m}\left(D, \varphi_{\max }, t\right)\right)$. It follows that $\mathfrak{a}\left(D, \varphi_{\max }, t\right)_{m} \subseteq \mathfrak{a}_{m}$ and $\varphi_{\max , t}^{D} \leq \varphi$. Therefore, $\varphi_{\max }=\lim _{t \rightarrow \infty} \varphi_{\max , t}^{D} \leq \varphi$, which forces $\varphi_{\max }=\varphi$.

For every nontrivial tempered valuation $v$, we define

$$
\sigma_{v}(\|D\|):=\lim _{\varepsilon \rightarrow 0^{+}} v(\|D+\varepsilon A\|)
$$

for some ample divisor $A$. Note that [Nakayama 2004] verifies that this definition is independent of the choice of the ample divisor $A$.

Proposition 6.11. The set $\mathrm{PSH}_{\sigma}(D)$ is closed under taking the supremum. The maximal pseudo D-psh function $\phi_{\max }$ can be expressed as $\phi_{\max }(v)=-\sigma_{v}(\|D\|)$ explicitly for all $v \in V_{X}^{*}$.

Proof. Let $\varphi_{\lambda}$ be a family of pseudo $D$-psh functions, and let $\varphi=\sup _{\lambda} \varphi_{\lambda}$. By Theorem 6.6 there exists an ample divisor $G$ such that $\varphi_{\lambda, k} \in \operatorname{PSH}\left(D+\frac{1}{k} G\right)$, where $\varphi_{\lambda, k}=\frac{1}{k} \log \left|\mathscr{F}\left(k \varphi_{\lambda}\right)\right|$. We have $\sup _{\lambda} \varphi_{\lambda, k} \in \operatorname{PSH}\left(D+\frac{1}{k} G\right)$ for every $k$ by Proposition 6.10. Since $\sum_{\lambda} \mathscr{g}\left(k \varphi_{\lambda}\right) \subseteq \mathscr{g}(k \varphi)$, we have $\varphi_{k} \geq \sup _{\lambda} \varphi_{\lambda, k} \geq \varphi$. Hence

$$
\varphi=\lim _{k \rightarrow \infty}\left(\sup _{\lambda} \varphi_{\lambda, k}\right) \in \operatorname{PSH}_{\sigma}(D) \text {. }
$$


Now we prove that $\phi_{\max }(v)=-\sigma_{v}(\|D\|)$ for all $v \in V_{X}^{*}$. Let $\phi(v)=-\sigma_{v}(\|D\|)$, and let $\varphi_{\max }^{\epsilon}$ be the maximal $(D+\epsilon A)$-psh function for every $\epsilon \ll 1$. We see that $\phi=\lim _{\epsilon \rightarrow 0^{+}} \varphi_{\max }^{\epsilon}$ pointwise. Because $\varphi_{\max }^{\epsilon}$ is decreasing as $\epsilon \rightarrow 0^{+}, \mathscr{f}\left(m \varphi_{\max }^{\epsilon}\right)$ form a descending chain of ideals as $\epsilon \rightarrow 0^{+}$for every integer $m>0$. If we fix an integer $m$ and a sequence $\epsilon_{1}>\epsilon_{2}>\cdots$ such that $\lim _{k \rightarrow \infty} \epsilon_{k}=0$, then the descending chain stabilizes when $k \gg 0$ because there exists an ample divisor $G$ such that $m D+G$ is Cartier and $\mathrm{O}_{X}(m D+G) \otimes \mathscr{F}\left(m \varphi_{\max }^{\epsilon_{k}}\right)$ is globally generated for every $k \gg 0$. It follows that $\left\|\varphi_{\max }^{\epsilon_{k}}-\varphi_{\max }^{\epsilon_{k^{\prime}}}\right\|<\frac{1}{m}$ for all sufficiently large $k$ and $k^{\prime}$. Equivalently, $\varphi_{\max }^{\epsilon_{k}}$ form a Cauchy sequence with respect to the norm. Therefore $\varphi_{\max }^{\epsilon_{k}}$ converges to $\phi$ strongly in norm, and hence $\phi \in \operatorname{PSH}_{\sigma}(D)$. Note that $\phi_{\max } \leq \varphi_{\max }^{\epsilon}$, and hence $\phi_{\max } \leq \phi$, which implies the conclusion.

Question 6.12 [Lehmann 2011, Question 6.15]. Is the maximal pseudo D-psh function algebraic?

Abundant divisors, introduced in [Nakayama 2004; Boucksom et al. 2013a], form a class of pseudo-effective divisors with nice asymptotic behaviour. We denote by $\kappa_{\sigma}(D)$ the numerical Kodaira dimension. A pseudo-effective divisor $D$ is said to be abundant if $\kappa(D)=\kappa_{\sigma}(D)$. We present the following easy corollary for the reader's convenience.

Corollary 6.13. (1) The set $\operatorname{PSH}(D)$ is nonempty if and only if $D$ is $\mathbb{Q}$-effective.

(2) $0 \in \operatorname{PSH}(D)$ if and only if $D$ is nef and abundant.

(3) The set $\mathrm{PSH}_{\sigma}(D)$ is nonempty if and only if $D$ is pseudo-effective.

(4) $0 \in \mathrm{PSH}_{\sigma}(D)$ if and only if $D$ is nef.

(5) Let $\varphi_{\max }$ be the maximal D-psh function, and $\phi_{\max }$ be the maximal pseudo $D$-psh function. Then, $D$ is abundant if and only if $\varphi_{\max }=\phi_{\max }$.

Proof. The first statement is trivial. The second is a consequence of the main result of [Russo 2009], and (4) follows from (2) immediately. If $D$ is not pseudo-effective, then $\mathrm{PSH}_{\sigma}(D)$ is empty from (1). We prove (3) as follows. If $D$ pseudo-effective, then $\mathrm{PSH}_{\sigma}(D)$ is nonempty by Proposition 6.11. To prove (5), simply notice that $D$ is abundant if and only if $v(\|D\|)=\sigma_{v}(\|D\|)$ for every divisorial valuation $v$ by [Lehmann 2011, Proposition 6.18] and the last statement follows by Propositions 6.10 and 6.11 .

Question 6.14. Assume that the divisor $D$ is abundant. Is the set $\operatorname{PSH}(D)$ equal to the set $\mathrm{PSH}_{\sigma}(D)$ ?

We introduce the following definition of the perturbed ideal and the diminished ideal as [Lehmann 2011, Definitions 4.3 and 6.2]. We use the notation $\mathscr{f}_{\sigma,-}(D)$ instead of $\mathscr{F}_{-}(D)$ to avoid that readers may confuse it with the notation $\mathscr{f}_{-}(\varphi)$. 
Definition 6.15. Let $D$ be a pseudo-effective divisor. In the finite descending chain $\left\{\mathscr{F}\left(\left\|L+\frac{1}{m} A\right\|\right)\right\}_{m=1}^{\infty}$, we define the perturbed ideal $\mathscr{F}_{\sigma,-}(D)$ to be the smallest

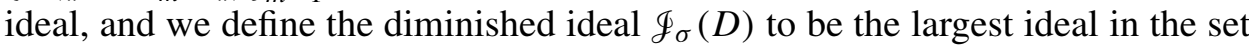
$\left\{\mathscr{F}_{\sigma,-}((1+\epsilon) D)\right\}_{\epsilon>0}$.

Finally, we obtain a generalization of [Lehmann 2011, Theorem 6.14].

Theorem 6.16. Let $D$ be a pseudo-effective divisor. Assume that $\phi_{\max }$ is the maximal pseudo D-psh function. Then, the perturbed ideal $\mathscr{F}_{\sigma,-}(D)=\mathscr{F}_{-}\left(\phi_{\max }\right)$, and the diminished ideal $\mathscr{F}_{\sigma}(D)=\mathscr{F}\left(\phi_{\max }\right)$. In particular, we can write $\mathscr{\Phi}_{\sigma}(D)$ explicitly as $\Gamma\left(U, \mathscr{\Phi}_{\sigma}(L)\right)=\left\{f \in \Gamma\left(U, \mathscr{O}_{X}\right) \mid v(f)+A(v)-\sigma_{v}(\|L\|)>0\right.$ for all $\left.v \in \mathrm{V}_{U}^{*}\right\}$. Further, a nonzero ideal $\mathfrak{q} \subseteq \mathscr{F}_{\sigma}(\|L\|)$ if and only if $v(\mathfrak{q})+A(v)-\sigma_{v}(\|L\|)>0$ for all $v \in \mathrm{V}_{X}^{*}$.

Proof. That $\mathscr{F}_{\sigma,-}(D)=\mathscr{F}_{-}\left(\phi_{\max }\right)$ follows from [Lehmann 2011, Proposition 4.7]. To prove the second equality, note that by definition $\mathscr{g}_{\sigma}(D)=\mathscr{I}\left((1+\epsilon) \varphi_{\max }^{\delta}\right)$, where $\varphi_{\max }^{\delta}$ denotes the maximal $(D+\delta A)$-psh function for an ample divisor $A$, sufficiently small $\epsilon$ and sufficiently small $\delta=\delta(\epsilon)$. From the proof of Proposition 6.11, $\varphi_{\max }^{\delta}$ converges to $\phi_{\max }$ strongly in norm. Therefore, Lemma 4.20 asserts that $\mathscr{F}\left(\phi_{\max }\right)=\mathscr{F}\left((1+\epsilon) \varphi_{\max }^{\delta}\right)=\mathscr{F}_{\sigma}(D)$ as $\delta \rightarrow 0^{+}$. The last statement is obvious by Corollary 5.8.

Remark 6.17. It should not be too difficult to generalize most results in this subsection from $\mathbb{Q}$-divisors to $\mathbb{R}$-divisors, that is, one can define $D$-psh functions for an $\mathbb{R}$-divisor $D$ and obtain similar results.

Finite generation. The goal of this subsection is to prove the finite generation proposition below as an application of qpsh functions. For definitions and properties of different types of Zariski decompositions, divisorial algebras and modules, we refer to [Nakayama 2004].

Proposition 6.18. Let $(X, B)$ be a log canonical pair. Assume that $K_{X}+B$ is $\mathbb{Q}$-Cartier and abundant, and that $R\left(K_{X}+B\right)$ is finitely generated. Then, for any reflexive sheaf $\mathscr{F}, M_{\mathscr{F}}^{p}\left(K_{X}+B\right)$ is a finitely generated $R\left(K_{X}+B\right)$-module.

Before we prove the proposition, we need a lemma.

Lemma 6.19 (global division). Let $X$ be a smooth projective variety of dimension $n$. Consider line bundles $L$ and $D$, a linear system $V \subseteq|L|$ spanned by the sections $\left\{s_{1}, \ldots, s_{l}\right\}$, and a D-psh function $\varphi$. If we denote by $\phi_{V}$ the $L$-psh function $\max _{1 \leq j \leq l} \log \left|s_{j}\right|$, then for every integer $m \geq n+2$, any section $\sigma$ in

$$
H^{0}\left(X, \mathcal{O}_{X}\left(K_{X}+m L+D\right) \otimes \mathscr{g}\left(m \phi_{V}+\varphi\right)\right)
$$

can be written as a linear combination $\sum_{j} s_{j} g_{j}$ of sections $g_{j}$ in

$$
H^{0}\left(X, \mathrm{O}_{X}\left(K_{X}+(m-1) L+D\right)\right) .
$$


Proof. Let $\left\{\varphi_{k} \in \mathscr{L}_{D}\right\}$ be a sequence of ideal functions that converges to $\varphi$ strongly in norm. Since $\mathscr{g}\left(m \phi_{V}+\varphi_{k}\right) \supseteq \mathscr{g}\left(m \phi_{V}+\varphi\right)$, the section $\sigma$ vanishes along the ideal $\mathscr{g}\left(m \phi_{V}+\varphi_{k}\right)$. If we denote by $\mathfrak{a}$ the base ideal $\mathfrak{b}(V)$, then $\phi_{V}=\log |\mathfrak{a}|$. Apply [Ein and Popa 2008, Theorem 4.1], and we deduce the conclusion.

Remark 6.20. In the statement of the theorem just cited, one can verify that the assumption that $D \otimes \mathfrak{b}^{\lambda}$ is nef and abundant implies that $\lambda \log |\mathfrak{b}|$ is $D$-psh. Note that Lemma 6.19 is not a generalization of the theorem because we did not obtain that every $g_{j}$ is in

$$
H^{0}\left(X, \mathrm{O}_{X}\left(K_{X}+(m-1) L+D\right) \otimes \mathscr{g}\left((m-1) \phi_{V}+\varphi\right)\right) .
$$

Nonetheless, it should be possible to generalize in the sense that

$$
g_{j} \in H^{0}\left(X, \mathscr{O}_{X}\left(K_{X}+(m-1) L+D\right) \otimes \mathscr{g}\left((m-1) \phi_{V}+\varphi\right)\right),
$$

if one can develop a theory on the restriction of qpsh functions to subvarieties (see the proof of [Ein and Popa 2008, Theorem 3.2]).

Proof of Proposition 6.18. We can assume that $(X, B)$ is $\log$ smooth of dimension $n$; $K_{X}+B$ is a $\mathbb{Q}$-Cartier $\mathbb{Q}$-divisor; and $\mathscr{F}=\mathcal{O}_{X}(A)$ is a very ample line bundle by [Birkar 2010, Theorem 1.1]. Since $R=R\left(K_{X}+B\right)$ is finitely generated, after a possible truncation we can assume that $R$ is generated by $R_{1}=H^{0}\left(m_{0}\left(K_{X}+B\right)\right)$ for some integer $m_{0}$ such that $m_{0}\left(K_{X}+B\right)$ is Cartier (see [ibid., Remarks 2.2 and 2.3]). If we set $\mathfrak{a}=\mathfrak{b}\left(\left|m_{0}\left(K_{X}+B\right)\right|\right)$ and $L:=m_{0}\left(K_{X}+B\right)$, then $\phi:=\log |\mathfrak{a}|$ is the maximal $L$-psh function. The rest of the proof is an analogue of [Demailly et al. 2013, Section 3]. Let $m$ be a sufficiently large integer (to be specified later), and let $\sigma$ be a global section of $m\left(K_{X}+B\right)+A$. We have

$$
m\left(K_{X}+B\right)+A=K_{X}+(n+2) L+D,
$$

where

$$
D:=B+\left(m-(n+2) m_{0}-1\right)\left(K_{X}+B+\frac{1}{m} A\right)+\frac{m_{0}(n+2)+1}{m} A .
$$

Set

$$
\varphi=\psi_{m}+\left(m-(n+2) m_{0}-1\right) \varphi_{m},
$$

where $\psi_{m}$ is $\left(B+\frac{1}{m}\left(m_{0}(n+2)+1\right) A\right)$-psh such that $\left\|\psi_{m}\right\|<1$, and $\varphi_{m}$ is the maximal $\left(K_{X}+B+\frac{1}{m} A\right)$-psh function. Notice that

$$
\|\log |\sigma|-(n+2) \phi-\varphi\|^{+} \leq\left\|\left(m_{0}(n+2)+1\right) \varphi_{m}-(n+2) \phi-\psi_{m}\right\|^{+} .
$$

We will show that $\left(m_{0}(n+2)+1\right) \varphi_{m} \leq(n+2) \phi$ for sufficiently large $m$, which implies that $\|\log |\sigma|-(n+2) \phi-\varphi\|^{+}<1$ and that by definition $\sigma$ vanishes along $\mathscr{S}((n+2) \phi+\varphi)$. Since $\phi$ is determined on some dual complex $\Delta(Y, D)$, it 
suffices to prove that $\left(m_{0}(n+2)+1\right) \varphi_{m} \leq(n+2) \phi$ on $\Delta(Y, D)$. Further, we can assume that $\phi$ is affine on $\Delta(Y, D)$. It suffices to check the inequality at vertices because $\varphi_{m}$ is convex on the dual complex. From the argument of Proposition 6.11, we see that $m_{0} \varphi_{m}$ converges to $\phi$ strongly in norm since $K_{X}+B$ is abundant. Therefore for sufficiently large $m$ the inequality $\left(m_{0}(n+2)+1\right) /(n+2) \varphi_{m} \leq \phi$ holds at vertices of $\Delta(Y, D)$, and hence for every nontrivial tempered valuation. Finally, $\sigma$ can be written as a linear combination $\sum_{j} s_{j} g_{j}$, where $g_{j}$ are sections in $H^{0}\left(X, \mathrm{O}_{X}\left(\left(m-m_{0}\right)\left(K_{X}+B\right)+A\right)\right.$ by Lemma 6.19 , which completes the proof.

Remark 6.21. The above finite generation proposition can be proved in another way as follows. Since the conclusion that $M_{\mathscr{F}}^{p}\left(K_{X}+B\right)$ is a finitely generated $R\left(K_{X}+B\right)$-module is equivalent to that $(X, B)$ has a good minimal model by [Birkar 2010, Theorem 1.3], it suffices to prove that $(X, B)$ has a good minimal model. By [Birkar and Hu 2012, Theorem 5.3] we conclude that $(X, B)$ has a log minimal model $\left(X^{\prime}, B^{\prime}\right)$. Since the positive part of the CKM-Zariski decomposition is semi-ample, the log minimal model $(X, B)$ is good. We here give a different proof without using the minimal model theory, in particular the length of extremal rays.

Proposition 6.18 can be slightly generalized as follows.

Definition 6.22. [Birkar et al. 2010, Definitions 3.6.4 and 3.6.6] Let $D$ be a divisor on $X$. A normal projective variety $Z$ is said to be the ample model of $D$ if there is a rational map $g: X \rightarrow Z$ and an ample $\mathbb{R}$-divisor $H$ on $Z$ such that if $p: W \rightarrow X$ and $q: W \rightarrow Z$ resolve $g$ then $q$ is a contraction and we can write $p^{*} D=q^{*} H+N$, where $N \geq 0$ is an $\mathbb{R}$-divisor and for every $B \sim_{\mathbb{Q}} p^{*} D$ then $B \geq N$. Let $(X, B)$ be a pair. A normal variety $Z$ is said to be the $\log$ canonical model of $(X, B)$ if it is the ample model of $K_{X}+B$.

Lemma 6.23. Let $D$ be an abundant divisor on a normal projective variety $X$. Assume that $D$ has the ample model. Then, $R(D)$ is finitely generated.

Proof. After replacing $X$ by a $\log$ resolution, we can assume that $g: X \rightarrow Z$ is a morphism and $D=P+N=g^{*} H+N$, where $H$ is an ample $\mathbb{R}$-divisor on the ample model $Z$ and $N \geq 0$ is an $\mathbb{R}$-divisor such that for every $B \sim_{\mathbb{Q}} D$ we have $B \geq N$. Note that $D=P+N$ is a CKM-Zariski decomposition. Since $D$ is abundant, we have that Fix $\|D\|=N_{\sigma}(D) \leq N \leq$ Fix $\|D\|$ by [Lehmann 2011, Proposition 6.18] and hence $P=P_{\sigma}(D)$. Furthermore, we can assume that there exist a smooth projective variety $T$ and a big $\mathbb{Q}$-divisor $G$ on $T$ such that $\mu: X \rightarrow T$ is a contraction and $P_{\sigma}(D)=P_{\sigma}\left(\mu^{*} G\right)$ by [Lehmann 2014, Theorems 5.7 and 6.1]. It follows that $Z$ is also the ample model of $G$. Notice that the rational map $h: T \rightarrow Z$ is birational. Therefore $H=p_{*} G$ is an $\mathbb{R}$-Cartier $\mathbb{Q}$-divisor and hence $\mathbb{Q}$-Cartier, which completes the proof. 
Finally, we obtain the proposition below by combining Proposition 6.18 and Lemma 6.23.

Proposition 6.24. Let $(X, B)$ be a log canonical pair. Assume that $K_{X}+B$ is $\mathbb{Q}$-Cartier and abundant, and that $(X, B)$ has the log canonical model. Then, $R\left(K_{X}+B\right)$ is finitely generated. Further, for any reflexive sheaf $\mathscr{F}_{F}, M_{\mathscr{F}}^{p}\left(K_{X}+B\right)$ is a finitely generated $R\left(K_{X}+B\right)$-module.

\section{Acknowledgements}

This paper is based on part of the author's $\mathrm{PhD}$ thesis. I would like to express my deep gratitude to my supervisor Professor Kefeng Liu for numerous conversations and encouragement from him. I am indebted to Professor Hongwei Xu and many other professors in Zhejiang University because I have greatly benefited from the courses and discussions with them. I would also like to thank Professor Sébastien Boucksom, Mattias Jonsson and Mircea Mustaţă for patiently answering my questions and providing many valuable comments. The author is supported by the EPSRC grant EP/I004130/1.

\section{References}

[Birkar 2010] C. Birkar, "Divisorial algebras and modules on schemes", preprint, 2010. To appear in J. Math. Soc. Japan. arXiv 1105.0441

[Birkar and $\mathrm{Hu}$ 2012] C. Birkar and Z. Hu, "Polarized pairs, log minimal models, and Zariski decompositions”, preprint, 2012. To appear in J. Math. Soc. Japan. arXiv 1302.4015

[Birkar et al. 2010] C. Birkar, P. Cascini, C. D. Hacon, and J. McKernan, "Existence of minimal models for varieties of log general type”, J. Amer. Math. Soc. 23:2 (2010), 405-468. MR 2011f:14023 Zbl 1210.14019

[Boucksom 2004] S. Boucksom, "Divisorial Zariski decompositions on compact complex manifolds", Ann. Sci. École Norm. Sup. (4) 37:1 (2004), 45-76. MR 2005i:32018 Zbl 1054.32010

[Boucksom et al. 2008] S. Boucksom, C. Favre, and M. Jonsson, "Valuations and plurisubharmonic singularities", Publ. Res. Inst. Math. Sci. 44:2 (2008), 449-494. MR 2009g:32068 Zbl 1146.32017

[Boucksom et al. 2012a] S. Boucksom, C. Favre, and M. Jonsson, "A refinement of Izumi's Theorem”, preprint, 2012. arXiv 1209.4104

[Boucksom et al. 2012b] S. Boucksom, C. Favre, and M. Jonsson, "Singular semipositive metrics in non-Archimedean geometry", preprint, 2012. arXiv 1201.0187

[Boucksom et al. 2012c] S. Boucksom, C. Favre, and M. Jonsson, "Solution to a non-Archimedean Monge-Ampère equation", preprint, 2012. arXiv 1201.0188

[Boucksom et al. 2012d] S. Boucksom, T. de Fernex, and C. Favre, "The volume of an isolated singularity", Duke Math. J. 161:8 (2012), 1455-1520. MR 2931273 Zbl 1251.14026

[Boucksom et al. 2013a] S. Boucksom, J.-P. Demailly, M. Păun, and T. Peternell, "The pseudoeffective cone of a compact Kähler manifold and varieties of negative Kodaira dimension", $J$. Algebraic Geom. 22:2 (2013), 201-248. MR 3019449 Zbl 1267.32017

[Boucksom et al. 2013b] S. Boucksom, T. de Fernex, C. Favre, and S. Urbinati, "Valuation spaces and multiplier ideals on singular varieties", preprint, 2013. arXiv 1307.0227 
[Demailly 1992] J.-P. Demailly, "Regularization of closed positive currents and intersection theory", J. Algebraic Geom. 1:3 (1992), 361-409. MR 93e:32015 Zbl 0777.32016

[Demailly 1993] J.-P. Demailly, "A numerical criterion for very ample line bundles", J. Differential Geom. 37:2 (1993), 323-374. MR 94d:14007 Zbl 0783.32013

[Demailly et al. 2000] J.-P. Demailly, L. Ein, and R. Lazarsfeld, "A subadditivity property of multiplier ideals", Michigan Math. J. 48 (2000), 137-156. Dedicated to William Fulton on the occasion of his 60th birthday. MR 2002a:14016 Zbl 1077.14516

[Demailly et al. 2013] J.-P. Demailly, C. D. Hacon, and M. Păun, "Extension theorems, non-vanishing and the existence of good minimal models", Acta Math. 210:2 (2013), 203-259. MR 3070567 Zbl 1278.14022

[Ein and Popa 2008] L. Ein and M. Popa, "Global division of cohomology classes via injectivity”, Michigan Math. J. 57 (2008), 249-259. Special volume in honor of Melvin Hochster. MR 2009k:14038 Zbl 1177.14043

[Ein et al. 2006] L. Ein, R. Lazarsfeld, M. Mustață, M. Nakamaye, and M. Popa, "Asymptotic invariants of base loci”, Ann. Inst. Fourier (Grenoble) 56:6 (2006), 1701-1734. MR 2007m:14008

[Ein et al. 2009] L. Ein, R. Lazarsfeld, M. Mustață, M. Nakamaye, and M. Popa, "Restricted volumes and base loci of linear series", Amer. J. Math. 131:3 (2009), 607-651. MR 2010g:14005

[Favre and Jonsson 2004] C. Favre and M. Jonsson, The valuative tree, Lecture Notes in Mathematics 1853, Springer, Berlin, 2004. MR 2006a:13008 Zbl 1064.14024

[Favre and Jonsson 2005a] C. Favre and M. Jonsson, "Valuations and multiplier ideals", J. Amer. Math. Soc. 18:3 (2005), 655-684. MR 2007b:14004 Zbl 1075.14001

[Favre and Jonsson 2005b] C. Favre and M. Jonsson, "Valuative analysis of planar plurisubharmonic functions”, Invent. Math. 162:2 (2005), 271-311. MR 2006k:32064 Zbl 1089.32032

[Jonsson and Mustață 2012] M. Jonsson and M. Mustaţă, "Valuations and asymptotic invariants for sequences of ideals", Ann. Inst. Fourier (Grenoble) 62:6 (2012), 2145-2209. MR 3060755

[Jonsson and Mustață 2014] M. Jonsson and M. Mustaţă, "An algebraic approach to the openness conjecture of Demailly and Kollár", J. Inst. Math. Jussieu 13:1 (2014), 119-144. MR 3134017

[Kollár and Mori 1998] J. Kollár and S. Mori, Birational geometry of algebraic varieties, Cambridge Tracts in Mathematics 134, Cambridge University Press, Cambridge, 1998. With the collaboration of C. H. Clemens and A. Corti, Translated from the 1998 Japanese original. MR 2000b:14018 Zbl 0926.14003

[Lazarsfeld 2004] R. Lazarsfeld, Positivity in algebraic geometry. II, Ergebnisse der Mathematik und ihrer Grenzgebiete. 3. Folge. A Series of Modern Surveys in Mathematics [Results in Mathematics and Related Areas. 3rd Series. A Series of Modern Surveys in Mathematics] 49, Springer, Berlin, 2004. Positivity for vector bundles, and multiplier ideals. MR 2005k:14001b Zbl 1093.14500

[Lehmann 2011] B. Lehmann, “Algebraic bounds on analytic multiplier ideals”, preprint, 2011. arXiv 1109.4452

[Lehmann 2014] B. Lehmann, “On Eckl's pseudo-effective reduction map”, Trans. Amer. Math. Soc. 366:3 (2014), 1525-1549. MR 3145741

[Nakayama 2004] N. Nakayama, Zariski-decomposition and abundance, MSJ Memoirs 14, Mathematical Society of Japan, Tokyo, 2004. MR 2005h:14015 Zbl 1061.14018

[Russo 2009] F. Russo, "A characterization of nef and good divisors by asymptotic multiplier ideals", Bull. Belg. Math. Soc. Simon Stevin 16:5, Linear systems and subschemes (2009), 943-951. MR 2011b:14021 Zbl 1183.14011 
Received February 1, 2012. Revised May 7, 2014.

ZHENGYU HU

DPMMS, CENTRE FOR MATHEMATICAL SCIENCES

CAMBRIDGE UNIVERSITY

WILBERFORCE ROAD

CAMBRIDGE, CB3 0WB

UNITED KINGDOM

zh262@dpmms.cam.ac.uk 


\title{
PACIFIC JOURNAL OF MATHEMATICS
}

\author{
msp.org/pjm
}

Founded in 1951 by E. F. Beckenbach (1906-1982) and F. Wolf (1904-1989)

\section{EDITORS}

Don Blasius (Managing Editor)

Department of Mathematics

University of California

Los Angeles, CA 90095-1555

blasius@math.ucla.edu

\author{
Paul Balmer \\ Department of Mathematics \\ University of California \\ Los Angeles, CA 90095-1555 \\ balmer@math.ucla.edu \\ Robert Finn \\ Department of Mathematics \\ Stanford University \\ Stanford, CA 94305-2125 \\ finn@math.stanford.edu \\ Sorin Popa \\ Department of Mathematics \\ University of California \\ Los Angeles, CA 90095-1555 \\ popa@math.ucla.edu
}

\author{
Vyjayanthi Chari \\ Department of Mathematics \\ University of California \\ Riverside, CA 92521-0135 \\ chari@math.ucr.edu \\ Kefeng Liu \\ Department of Mathematics \\ University of California \\ Los Angeles, CA 90095-1555 \\ liu@math.ucla.edu \\ Jie Qing \\ Department of Mathematics \\ University of California \\ Santa Cruz, CA 95064 \\ qing@ cats.ucsc.edu
}

\section{PRODUCTION}

Silvio Levy, Scientific Editor, production@msp.org

\section{SUPPORTING INSTITUTIONS}

ACADEMIA SINICA, TAIPEI

CALIFORNIA INST. OF TECHNOLOGY

INST. DE MATEMÁTICA PURA E APLICADA

KEIO UNIVERSITY

MATH. SCIENCES RESEARCH INSTITUTE

NEW MEXICO STATE UNIV.

OREGON STATE UNIV.

\author{
STANFORD UNIVERSITY \\ UNIV. OF BRITISH COLUMBIA \\ UNIV. OF CALIFORNIA, BERKELEY \\ UNIV. OF CALIFORNIA, DAVIS \\ UNIV. OF CALIFORNIA, LOS ANGELES \\ UNIV. OF CALIFORNIA, RIVERSIDE \\ UNIV. OF CALIFORNIA, SAN DIEGO \\ UNIV. OF CALIF., SANTA BARBARA
}

\author{
Daryl Cooper \\ Department of Mathematics \\ University of California \\ Santa Barbara, CA 93106-3080 \\ cooper@math.ucsb.edu \\ Jiang-Hua Lu \\ Department of Mathematics \\ The University of Hong Kong \\ Pokfulam Rd., Hong Kong \\ jhlu@maths.hku.hk \\ Paul Yang \\ Department of Mathematics \\ Princeton University \\ Princeton NJ 08544-1000 \\ yang@math.princeton.edu
}

These supporting institutions contribute to the cost of publication of this Journal, but they are not owners or publishers and have no responsibility for its contents or policies.

See inside back cover or msp.org/pjm for submission instructions.

The subscription price for 2014 is US $\$ 410 /$ year for the electronic version, and \$535/year for print and electronic.

Subscriptions, requests for back issues and changes of subscribers address should be sent to Pacific Journal of Mathematics, P.O. Box 4163, Berkeley, CA 94704-0163, U.S.A. The Pacific Journal of Mathematics is indexed by Mathematical Reviews, Zentralblatt MATH, PASCAL CNRS Index, Referativnyi Zhurnal, Current Mathematical Publications and Web of Knowledge (Science Citation Index).

The Pacific Journal of Mathematics (ISSN 0030-8730) at the University of California, c/o Department of Mathematics, 798 Evans Hall \#3840, Berkeley, CA 94720-3840, is published twelve times a year. Periodical rate postage paid at Berkeley, CA 94704, and additional mailing offices. POSTMASTER: send address changes to Pacific Journal of Mathematics, P.O. Box 4163, Berkeley, CA 94704-0163.

PJM peer review and production are managed by EditFLOW ${ }^{\circledR}$ from Mathematical Sciences Publishers.

\section{PUBLISHED BY}

\section{mathematical sciences publishers \\ nonprofit scientific publishing}

http://msp.org/

(C) 2014 Mathematical Sciences Publishers 


\section{PACIFIC JOURNAL OF MATHEMATICS}

Volume $270 \quad$ No. $1 \quad$ July 2014

Hermitian categories, extension of scalars and systems of sesquilinear forms

EVA BAYER-FLUCKIGER, URIYA A. FIRST and DANIEL A.

MOLDOVAN

Realizations of the three-point Lie algebra $\mathfrak{s l}(2, \mathscr{R}) \oplus\left(\Omega_{\mathscr{R}} / d \mathscr{R}\right)$

BEN COX and ELIZABETH JURISICH

Multi-bump bound state solutions for the quasilinear Schrödinger equation with critical frequency

YUXIA GUO and ZHONGWEI TANG

On stable solutions of the biharmonic problem with polynomial growth

Hatem Hajlaoui, Abdellaziz Harrabi and Dong Ye

Valuative multiplier ideals

ZHENGYU HU

Quasiconformal conjugacy classes of parabolic isometries of complex hyperbolic space

YOUNGJU KIM

On the distributional Hessian of the distance function

Carlo MantegazZa, Giovanni Mascellani and Gennady URALTSEV

Noether's problem for abelian extensions of cyclic $p$-groups

IVO M. MICHAILOV

Legendrian $\theta$-graphs

Danielle O’Donnol and Elena PaVelescu

A class of Neumann problems arising in conformal geometry

WEIMIN SHENG and LI-XIA YUAN

Ryshkov domains of reductive algebraic groups

TAKAO WATANABE 